\title{
Broad Emission Line Shifts in Quasars: An Orientation Measure for Radio-Quiet Quasars?
}

\author{
Gordon T. Richards ${ }^{1}$, Daniel E. Vanden Berk ${ }^{2}$, Timothy A. Reichard ${ }^{1}$, Patrick B. Hall ${ }^{3,4}$, Donald \\ P. Schneider ${ }^{1}$, Mark SubbaRao ${ }^{5}$, Anirudda R. Thakar ${ }^{6}$, and Donald G. York ${ }^{5,7}$
}

\begin{abstract}
Using a sample of 3814 quasars from the Early Data Release of the Sloan Digital Sky Survey, we confirm that high-ionization, broad, emission lines such as C IV are significantly blueshifted with respect to low-ionization, broad, emission lines, such as Mg II, which are thought to be close to the systemic redshift. We examine the velocity shifts of the Mg II and C IV emission lines with respect to [O III] and Mg II, respectively. C IV emission line peaks have a range of shifts from a redshift of $500 \mathrm{~km} \mathrm{~s}^{-1}$ to blueshifts well in excess of $2000 \mathrm{~km} \mathrm{~s}^{-1}$ as compared to $\mathrm{Mg}$ II. We confirm previous results that suggest an anti-correlation between the shift of the C IV emission line peak and the rest equivalent width of the C IV emission line. Furthermore, by creating composite quasar spectra as a function of C IV shift, we are able to study, in detail, the profiles of the line as a function of velocity shift. We find that the apparent shift of the C IV emission line peak is not a shift so much as it is a lack of flux in the red wing for the composite with the largest apparent shift. This observation should strongly constrain models for the broad emission line region in quasars. The emission line blueshift and equivalent width of C IV are also discussed in light of the well-known anti-correlation between the equivalent width of C IV emission and continuum luminosity, otherwise known as the Baldwin Effect. We further discuss the C IV emission line shift as a function of other quasar properties such as spectral index, radio and X-ray detection. We find a possible correlation between the $\mathrm{C}$ IV emission line shifts and the radio properties of the quasars that is suggestive of orientation as the cause of the C IV velocity shifts. Finally, we explore whether the C IV emission line blueshifts correlate with the presence of broad
\end{abstract}

\footnotetext{
${ }^{1}$ Department of Astronomy and Astrophysics, The Pennsylvania State University, University Park, PA 16802

${ }^{2}$ Fermi National Accelerator Laboratory, P.O. Box 500, Batavia, IL 60510

${ }^{3}$ Princeton University Observatory, Princeton, NJ 08544

${ }^{4}$ Pontificia Universidad Católica de Chile, Departamento de Astronomía y Astrofísica, Facultad de Física, Casilla 306, Santiago 22, Chile

${ }^{5}$ The University of Chicago, Department of Astronomy and Astrophysics, 5640 S. Ellis Ave., Chicago, IL 60637

${ }^{6}$ Department of Physics and Astronomy, The Johns Hopkins University, 3701 San Martin Drive, Baltimore, MD 21218

${ }^{7}$ The University of Chicago, Enrico Fermi Institute, 5640 S. Ellis Ave., Chicago, IL 60637
} 
absorption line absorption troughs or with narrow, "associated" absorption and how these might be related to orientation.

Subject headings: quasars: emission lines — quasars: general — line: formation — line: profiles

\section{Introduction}

It has long been known that the redshifts derived from different quasar emission lines often do not agree with each other within typical measurement errors. Gaskell (1982) performed the first detailed study of this phenomenon and concluded that high-ionization, broad, emission lines such as C IV are shifted by a few hundred $\mathrm{km} \mathrm{s}^{-1}$ blueward of the redshifts determined from low-ionization, broad, emission lines such as Mg II. Subsequent studies (Wilkes 1984; Espey et al. 1989; Corbin 1990; Tytler \& Fan 1992; McIntosh et al. 1999) have confirmed these shifts beyond any shadow of a doubt. Using a sample of over 2200 active galactic nuclei (AGN), Vanden Berk et al. (2001) demonstrated that these effects are also present in the sample of quasars from the Sloan Digital Sky Survey (SDSS;York et al. 2000).

These shifts affect many of the most important issues in quasar-related science (both directly and indirectly). The most obvious area of research that is affected is in the modeling of the phenomena of AGN, particularly the broad emission line region (BELR; Peterson 1997; Krolik 1999). BELR models based upon accretion disk winds (Murray \& Chiang 1997; Proga, Stone, \& Kallman 2000) will have different dynamics than cloud-based models (Blumenthal \& Mathews 1975; Capriotti, Foltz, \& Byard 1980). These differences affect the line profiles and their locations in ways that should be testable.

In addition to investigations of the BELR, investigations regarding associated absorption (Foltz et al. 1986), broad absorption lines (Weymann et al. 1991), and the cosmic UV and X-ray background are affected by the apparent blueshift of the C IV emission line. We will comment on each of these issues in turn.

Determining the nature of the so-called "associated absorber" (Foltz et al. 1986) population $\left(z_{\mathrm{abs}} \approx z_{\mathrm{em}}\right)$, requires understanding the velocity shift of the C IV emission line in quasars. Some associated absorbers are observed to have redshifts greater than the quasar redshift as determined from the C IV emission line peak. However, as many authors have suggested, these apparent

redshifts could simply be the result of the blueshift of the $\mathrm{C}$ IV emission line relative to the systemic redshift of the quasar. Given the average observed blueshift of the C IV emission line, it is clear that the population of truly $z_{\mathrm{abs}} \geq z_{\mathrm{em}}$ systems is smaller than it would appear, but it remains unclear if these systems can be completely explained as outflows that only appear to be infalling because of the observed shift of the C IV emission line peak.

In addition to the narrower associated absorbers, emission line shifts are important for broad 
absorption line (BAL) quasars. Previous investigations of the velocity shifts of quasar emission lines, such as that of Corbin (1990), suggest a correlation between the velocity shift of the emission lines and the broad absorption line troughs observed in at least $10 \%$ of all quasars (Weymann 1997). However, Weymann et al. (1991) saw no such effect. Whether or not the velocity shifts are an orientation effect has strong implications for BAL quasars. If we can determine the nature of the emission line shifts, we can likely settle the issue of how BALs are distributed relative to the plane of the accretion disk that is thought to feed AGN. Are BAL flows found preferentially in the plane of the accretion disk, or are the flows more spherically symmetric in their distribution?

In addition to the more obvious problems associated with our inability to determine accurate redshifts for quasars, we must also consider the effect that the apparent inconsistency of emission line redshifts has on indirect quasar science. For example, the blueshift of the C IV emission line causes an over-estimation of the ionizing flux in the inter-galactic medium, such as is described by McIntosh et al. (1999).

The issue of C IV emission blueshift takes on even more importance if these shifts are correlated with the line of sight orientation of quasars, or with an orientation-type parameter such as the opening angle of a disk-wind. The most obvious ramification of such a possibility is the ability to determine orientation angles for radio-quiet quasars: until now, only a small percentage of quasars with distinct radio properties could be analyzed in terms of orientation. If a measure of orientation for radio-quiet quasars could be found and is robust (whether it be related to the C IV emission line shifts or not), it would likely revolutionize the study of quasars.

The format of the paper is as follows. In $\S 2$, we describe the data used in this analysis. Throughout this paper we will follow Schneider et al. (2002) and use a cosmology where $\mathrm{H}_{\mathrm{o}}=50 \mathrm{~km} \mathrm{~s}^{-1} \mathrm{Mpc}^{-1}, \Omega=1, \Lambda=0$. We also adopt a quasar-centered coordinate system in which outflows from the quasar along our line of sight (which appear blueshifted) have positive velocities. Section 3 presents an analysis of the data in terms of composite quasar spectra, and the radio and X-ray properties of our sample. In $\S 4$, we discuss the implications of our findings for modeling of the BELR, the Baldwin Effect, the origin of intrinsic absorption, and orientation measures. We also give suggestions for future work. Finally, $\S 5$ presents a summary of our results.

\section{Data}

The quasars were selected for spectroscopic followup from the SDSS imaging survey which uses a wide-field multi-CCD camera (Gunn et al. 1998); quasar candidates were identified using a preliminary version of the SDSS Quasar Target Selection Algorithm (Richards et al. 2002). Objects were selected based on both their broad-band SDSS colors (Fukugita et al. 1996; Stoughton et al. 2002) and as optical matches to radio-detected quasars from the VLA "FIRST" survey (Becker, White, \& Helfand 1995). All of the emission line properties are taken directly from the automated 1-D spectroscopic pipeline (SPECTRO; Frieman et al., unpublished; Stoughton et al. 2002, § 4.10) 
outputs that are stored in the public database.

We study a sample of 3814 bona-fide quasars $\left(M_{i}<-23\right.$, with at least one line broader than $1000 \mathrm{~km} \mathrm{~s}^{-1}$ ) from the Early Data Release (EDR; Stoughton et al. 2002) quasar sample (Schneider et al. 2002). Though the sample is not technically homogeneous, it is adequate for the scientific goals of this work. The differences between the EDR versions of quasar target selection (Stoughton et al. 2002) and the final version of quasar target selection (Richards et al. 2002) are largely for $z>2.2$, whereas our sample is restricted to $z \leq 2.2$. In addition, we cannot think of any reason why the EDR quasar sample would be biased against (or towards) quasars with large (or small) emission line blueshifts.

Two sub-samples of quasars were created, one for the study of the velocity shifts between C IV and Mg II, the other for the study of the velocity shifts between Mg II and [O III]. These samples contain 794 and 417 quasars, respectively.

For the Mg II - [O III] sample we take the redshift determined from [O III] to be the systemic (center of mass) redshift, since previous studies have shown that the redshifts determined from narrow forbidden lines agree well with redshifts determined from stellar absorption features (Vrtilek \& Carleton 1985) and H I $21 \mathrm{~cm}$ emission (Hutchings, Gower, \& Price 1987) in the host galaxies of AGN; see the discussions in Tytler \& Fan (1992) and McIntosh et al. (1999). To ensure that both the $\mathrm{Mg}$ II and [O III] emission lines are in each of the spectra considered, the redshifts were restricted to $0.415 \leq z \leq 0.827$.

For the C IV - Mg II sample, our spectra do not cover [O III] and we take the redshift determined from Mg II to be the systemic redshift, since low-ionization broad lines such as Mg II have been shown to have a smaller velocity offset from the forbidden narrow lines than do highionization broad lines (e.g., Tytler \& Fan 1992). In this sample, the redshifts were restricted to $1.54 \leq z \leq 2.2$. These redshift restrictions ensure that both the $\mathrm{C}$ IV and $\mathrm{Mg}$ II emission lines are observed in each of the spectra considered (which span the range from $3800 \AA$ to $9200 \AA$ at a resolution of $\approx 1800$ ). The sample was further restricted by removing all broad absorption line quasars, since the BAL troughs hinder accurate measurement of the line peaks. BAL quasars were defined according to the computation of the traditional Weymann et al. (1991) "balnicity index" using the composite quasar spectrum from Vanden Berk et al. (2001) to define the continuum level. This list of BAL quasars is not yet final; some weaker BALs may move into or out of the sample. However, all strong BALs (balnicity $\gtrsim 500 \mathrm{~km} \mathrm{~s}^{-1}$ ) should be accounted for. Since the classification of weak BALs is subjective anyway, we have proceeded with our analysis using our current list of BALs.

The "systemic" redshifts used in this analysis were determined using only the [O III] or Mg II emission lines, respectively. Since our analysis is dependent upon how SPECTRO measures the peaks of these lines, we describe some details of the process. SPECTRO measures two sets of line parameters. The first are detected peaks in the spectrum which best match a list of common emission lines. Once an object's redshift has been determined, either from the detected emission 
lines or from cross-correlation with template spectra, a second set of line measurements are made at the expected positions of a more comprehensive set of spectral features. In both cases a single Gaussian is fit to the line. The first set are identified in the database as found and the second as measured.

The following concerns the measured line parameters, which are used in this paper. The code determines which lines are to be fit individually and which are to be fit as blends. The width, height and center of the line or blend of lines are then fit by a modified Levenberg-Marquardt algorithm. To enhance the robustness of the code, only a single shift from the expected positions is used for blended lines. The shift of the line center from the expected position is constrained to be $\pm 2000 \mathrm{~km} \mathrm{~s}^{-1}$ by multiplying the $\chi^{2}$ values by a steep ramp function beyond this range. In some cases the expected positions are not the redshifted laboratory wavelengths, but the redshifted average line positions as determined by the composite SDSS quasar spectrum (Vanden Berk et al. 2001); the line wavelengths relevant here are C IV $\lambda 1545.86$ and Mg II $\lambda 2800.32$ (which are doublets, but quasar emission line widths are broad enough that the doublet is not resolved). These wavelengths are used to compute the redshifts as reported in the SDSS database. In this analysis, we will determine the redshift for each individual line based on the SPECTRO measured line peaks, but we will use the laboratory rest-frame wavelengths (see Vanden Berk et al. 2001) as the expected line positions.

\section{Analysis}

\subsection{Distribution of Velocity Shifts}

The velocity distribution of C IV emission line shifts with respect to Mg II is quite large - as can be seen in Figure $1^{8}$. We find the shifts by taking the measured line peaks for C IV and Mg II from the SDSS database and using the laboratory rest-frame wavelengths of the C IV and $\mathrm{Mg}$ II doublets, (1549.06 and $2798.75 \AA$, respectively) to determine the redshift of each line. The median of the distribution is found to be $824 \mathrm{~km} \mathrm{~s}^{-1}$ with a dispersion of $\pm 511 \mathrm{~km} \mathrm{~s}^{-1}$. For individual quasars, the error in the C IV blueshift with respect to $\mathrm{Mg}$ II can be $\sim 500 \mathrm{~km} \mathrm{~s}^{-1}(2 \sigma)$. Spot checking of good quality spectra to determine the blueshift by hand supports this conclusion. We do not believe that this error is skewed towards either positive or negative shifts, so the errors in the ensemble average should be much lower. In general, the errors will be biggest for those quasars with the largest C IV blueshifts, since we find that those quasars have the weakest C IV emission lines; see $\S 3.2 .2$ for a more detailed discussion regarding errors.

In a similar manner to the C IV - Mg II shift, Figure 2 shows the distribution of velocity differences between the $\mathrm{Mg}$ II and [O III] emission line peaks. Again, we use the measured line

\footnotetext{
${ }^{8}$ Recall that, in this paper, positive velocities refer to blueshifts with respect to the quasar rest frame, whereas negative velocities indicate redshifts.
} 
peaks from the SDSS database, and the laboratory wavelengths of Mg II doublet and the reddest of the [O III] triplet (2798.75 and 5008.24 $\AA$, respectively) in order to determine the redshift of each emission line and the blueshift between the emission lines. The peak of this distribution is near zero, but has an overall median shift of $-97 \mathrm{~km} \mathrm{~s}^{-1}$ with a spread of $269 \mathrm{~km} \mathrm{~s}^{-1}$. This shift is about half as large as that found by Vanden Berk et al. (2001), who found a velocity offset of $-161 \mathrm{~km} \mathrm{~s}^{-1}$ for $\mathrm{Mg} \mathrm{II}{ }^{9}$; Tytler \& Fan (1992) found ambiguous results for the velocity shift of the Mg II emission line $\left(200 \mathrm{~km} \mathrm{~s}^{-1}\right.$ and $-380 \mathrm{~km} \mathrm{~s}^{-1}$ with respect to [O II] for two different samples, respectively). In our sample, the $\mathrm{Mg}$ II emission line, while quite close to what is thought to be the systemic redshift in the ensemble average, can have significant deviations in individual quasars (modulo measurement errors).

Other authors (e.g., McIntosh et al. 1999) have also studied the shift of the $\mathrm{H} \beta$ emission line. We are not able to do so using the automated reductions of the SDSS spectra. The SPECTRO pipeline treats $\mathrm{H} \beta$ and the nearby [O III] triplet as a blend with the same redshift (fortunately, redshifts determined from $\mathrm{H} \beta$ are typically similar to redshifts determined from [O III]). An analysis of the shift of the $\mathrm{H} \beta$ emission line with respect to [O III] would thus require a substantial modification to the SPECTRO pipeline and a reanalysis of all of the spectra: a task which is well beyond the scope of this paper.

\subsection{Composite Spectra}

\subsubsection{Sub-sample Definition}

To assess the differences between the spectra of quasars with both large and small C IV - Mg II redshift differences, we created a set of four composite quasar spectra by combining the spectra of quasars with similar velocity shifts. We first sorted the list of quasars according to their C IV $\mathrm{Mg}$ II redshift differences and then divided this list into four roughly equal lists of $\sim 200$ quasars each. Composite quasar spectra were created by combining the spectra (using the geometric mean, which is appropriate for power-law spectra) in each of these four samples following the procedure of Vanden Berk et al. (2001). Table 1 gives the velocity offset properties of these samples (denoted A, $\mathrm{B}, \mathrm{C}$, and D); it includes the number of objects (the number within the FIRST survey area is given in parentheses), the average velocity offset of the C IV emission line in the composite spectrum, along with the average, minimum and maximum velocity offsets of the $\mathrm{C}$ IV emission line in the individual spectra. Table 2 gives other properties of the samples, including the average redshift, the average absolute magnitude in the $i$-band, the average spectral index $\left(f_{\nu} \propto \nu^{\alpha}\right)$ of the optical continuum, the number and fraction of radio and X-ray detected sources, along with the equivalent width and the full-width at half-maximum of the C IV emission line (in $\AA$ ).

\footnotetext{
${ }^{9}$ Note the sign difference between this paper and Vanden Berk et al. (2001). The $+161 \mathrm{~km} \mathrm{~s}^{-1}$ quoted by Vanden Berk et al. (2001) becomes $-161 \mathrm{~km} \mathrm{~s}^{-1}$ in the notation used herein.
} 


\subsubsection{Velocity Errors}

We have binned the data into four different samples, in part, to minimize the effect of any errors in the emission line blueshift determination. Errors can cause objects move from one bin to the next, but it is unlikely that many objects from sample A will move into sample D (and vice versa) as a result of errors. Also, that the offset between the peaks of the Mg II emission lines for the composite spectra in $\S 3.2 .3$ is small suggests that the errors in the blueshift measurements are not a problem. Finally, we have measured the blueshift of C IV by hand (using the mode of the upper $50 \%$ of the line) in each of the composites. We find that the blueshifts are 197,606,1003, and $1526 \mathrm{~km} \mathrm{~s}^{-1}$, for composites A, B, C, and D, respectively. These values are in excellent agreement with the average blueshifts of the individual quasars that contribute to each composite as can be seen by comparing columns 3 and 4 in Table 1, thus we are confident that measurement errors do not significantly effect our results.

Note that, as discussed above, the measured emission line centers have an upper limit of $2000 \mathrm{~km} \mathrm{~s}^{-1}$ for the velocity difference of the line center from the accepted redshift of the quasar. Some of the C IV peaks are shifted by more than this $2000 \mathrm{~km} \mathrm{~s}^{-1}$, thus there is a "pile-up" at this velocity in the database. Future versions of SPECTRO will allow for shifts as large as $3000 \mathrm{~km} \mathrm{~s}^{-1}$. For those quasars affected by this limitation in SPECTRO (28 in all), we have measured the velocity shifts by hand; the C IV emission line peak was determined using the mode of the upper $50 \%$ of the line as was the case in Vanden Berk et al. (2001). The dashed histogram in Figure 1 shows the hand calculated velocity shifts of these quasars. We find that the error on these C IV shifts can be quite large and that the true upper velocity limit may be as high as or higher than $3000 \mathrm{~km} \mathrm{~s}^{-1}$.

\subsubsection{Composite Spectra Analysis}

In Figure 3, we plot the spectra of the composites whose inputs had the smallest and largest C IV emission line shifts, which we hereafter refer to as composites A and D, respectively. We also plot power-law continua with $\alpha=-0.392$ and a slightly bluer $\alpha=-0.297$ for composites A and $\mathrm{D}$, respectively to guide the eye. The spectral indices were computed using a range of $10 \AA$ at both $1355 \AA$ and $2200 \AA$. Neither of these ranges are ideal continuum windows, see Vanden Berk et al. (2001), but these are appropriate values to use in the available wavelength range. The composite spectra do not extend far enough into the red to use a better continuum window. For comparison, we have also created a composite quasar spectrum from all of the quasars in the EDR quasar catalog (Schneider et al. 2002), which differs from the sample used by (Vanden Berk et al. 2001). The EDR quasar composite has a spectral index of $\alpha=-0.410$ when measured in the manner described above. Note, however, that BALs, which may tend to be redder than average, are not excluded from the EDR composite quasar spectrum. Since these continua are clearly not optimal over the entire spectrum, we will use local continua for any further analysis.

Figure 4 shows the major emission line regions for all four of the (normalized) composite spectra 
(A, B, C, and D) with the red spectrum having the smallest C IV shift (composite A) and the blue spectrum showing the largest C IV shift (composite D). Here we have chosen to normalize the spectra in local continuum regions. The continuum regions $\left[\left(\lambda_{\min }: \lambda_{\max }\right)\right.$, in $\left.\AA\right]$ are $(1285: 1355)$, (1285:1355), (1355:1465), (1465:1695), (1830:1975), (2695:2955) for Lyman- $\alpha$, O I, Si IV, C IV, C III], and Mg II, respectively.

Of particular interest in Figure 4 is the profile of the C IV emission line as a function of velocity shift. By simple visual inspection, we confirm previous results that found an anti-correlation between the C IV emission line velocity shift and equivalent width (Corbin 1990). Furthermore, after the spectra have been normalized by the continuum, it is clear that the shift of the C IV emission line is not so much a shift of the centroid to the blue as it appears to be a suppression of flux in the red wing. The blue wing is quite similar in all four composite spectra, whereas the peak and the red wing are less pronounced as the velocity shift increases. We note that this conclusion depends significantly upon our continuum placement; however, we feel that our procedure is appropriate; see below for further discussion.

This extinction in the red wing coupled with the similarity of the blue wings is more complex than is generally reported. However, such an effect is not entirely without precedent: both Francis \& Koratkar (1995) and Corbin \& Boroson (1996) found enhanced Baldwin Effects (Baldwin 1977, also see $\S 4.2$ ) in the red wing of C IV. That the blueshift is apparently not a blueshift but rather a deficit of flux in the red wing significantly changes the interpretation of the C IV emission line shifts. The shift is clearly not the result of an actual change in the (apparent) bulk velocity flow (i.e., it is not a blueward shift of the entire line profile); instead, the shift may be caused entirely by obscuration or suppression of the lowest (or most negative) velocity gas (in the quasar rest frame), since the blue wings of both the small and large blueshift composites are co-located.

By dividing rather than subtracting the continuum, we implicitly assume that the flux in the emission lines is correlated with the continuum luminosity of the quasars, as expected since the BELR is powered primarily by photoionization induced by the UV continuum. This correlation is not perfect; higher-luminosity quasars have lower equivalent width emission lines (the Baldwin Effect, Baldwin 1977). However, the continuum luminosity of quasars has a range of values at least an order of magnitude larger than the range of emission line equivalent widths; for example, see Baldwin, Wampler, \& Gaskell (1989), Figure 5 and Krolik (1999), Figure 10.2. If the continuum was completely decoupled from the lines, the range of values spanned by emission line EQWs would be the larger of the two, since it would include intrinsic scatter plus uncorrelated variations produced by different continuum levels. As the opposite is true, dividing by the continuum is more appropriate than subtracting.

However, it could be argued that for some cases, such as when trying to understand the line profiles, it would be more appropriate to subtract the continuum. Thus, in Figure 5, we show the profiles of C IV and Mg II after subtracting the continuum and scaling the emission lines to have the same peak strength. Figure 5 demonstrates that the $\mathrm{C}$ IV line profile is relatively symmetric 
in composite $\mathrm{A}$, but is quite asymmetric in composite $\mathrm{D}$, which has more blue flux than red flux as compared to composite A (see also Figure 6). Note, however, that the scaling used distorts the relative fluxes between the composites; although composite D has more blue flux than red as compared to composite A, composite D has less overall blue flux (see Figure 4). In any case, we emphasize that the interpretation of the C IV profiles does depend significantly on how one handles the continuum.

By visual inspection, we find that our composites are similar to those created by Wills et al. (1993) except that here we divide the sample by their C IV - Mg II redshift differences instead of by the FWHM of the C IV emission line. In addition, we determine the redshift solely from the $\mathrm{Mg}$ II emission line. It is therefore interesting that we see many of the same properties as did Wills et al. (1993), such as the anti-correlation between the rest equivalent width (EQW) and full-width at half maximum (FWHM) of the C IV emission lines. Although the rest equivalent widths of the C IV emission lines are anti-correlated with the FWHM of the C IV emission lines in our samples (see Table 2), it is not clear that composite $\mathrm{D}$, in fact, has the broadest lines. If the $\mathrm{C}$ IV emission line profile is indeed absorbed in composite D as compared to composite A, then the FWHM of the emission line is largely unrelated to the velocity distribution of the emission line gas.

We further compare to Wills et al. (1993) by plotting, in Figure 6, the C IV profiles of each of our composites on top of reflections about the peaks of each of the $\mathrm{C}$ IV profiles. In these plots we have normalized the continua using the same windows as for C IV above; we find no qualitative difference in the inverted profiles if instead we use (1480:1620) Angstroms as the continuum window. The profiles are inverted around the observed peaks of the lines and not the expected line centers for C IV. If we used the laboratory wavelength as the reflection point, the asymmetries in the more blueshifted composites would be even more dramatic. Whereas Wills et al. (1993) find that their intermediate spectra are the most asymmetric, we find that the asymmetry in our spectra increases with increasing blueshift of the line peak.

It is also possible to create difference spectra in the vicinity of $\mathrm{C} I V$, similar to those made by Brotherton et al. (1994); these are shown in Figure 7. These spectra have been normalized as above; the difference between composite $\mathrm{A}$ and $\mathrm{D}$ is shown in black, $\mathrm{A}$ minus $\mathrm{C}$ in dark grey, and $\mathrm{A}$ minus $\mathrm{B}$ in light grey. Note that the red flux missing in composites B, C, and D extends to at least $1600 \AA$ - nearly $10,000 \mathrm{~km} \mathrm{~s}^{-1}$ from the line center. These C IV difference profiles are considerably more asymmetric than those of Brotherton et al. (1994). This difference is probably the result of our use of the Mg II redshift to define the systemic redshift. If instead we aligned the peaks of the C IV emission line in the composite spectra before we created the difference spectra, we would get more symmetric difference spectra. If the two component BELR model discussed by Wills et al. (1993) is valid, then these C IV difference spectra and the asymmetries of the line profiles place significant constraints upon the model; given the shape of the difference spectra, we would prefer an obscured or suppressed disk-wind outflow as the preferred explanation for the BELR (see $\S 4$ ).

Emission lines other than C IV are equally worthy of consideration. In particular, we note 
that, in Figure 4, the C III] profile seems to be shifted significantly blueward in composite D. This observation is consistent with the findings of Espey et al. (1989) and Corbin (1990). However, instead of interpreting this as a real shift of C III], we interpret this as an apparent shift due to an increase in the relative strength of Si III] $\lambda 1892$ (see Vanden Berk et al. 2001) versus C III], or simply perhaps as a weakening of C III]. Alternatively, the changes could be due to Fe III (UV34); see Vestergaard \& Wilkes (2001), § 5.2. If the interpretation that the change in line profile results from a change in the relative strength of the lines is correct, the velocity difference for the C III] peaks between composites $\mathrm{A}$ and $\mathrm{D}$ appears to be zero within the measurement errors (however, the errors are quite large given the severity of the blending). In any case, it may still be possible to make use of this emission feature, since, for the purpose of measuring the shifts, it matters less why the line shifts than the fact that it shifts in the first place.

The Lyman $-\alpha+\mathrm{N}$ V blend in the upper left-hand panel of Figure 4 is also of interest. The most obvious result is that the Lyman- $\alpha$ line is much stronger for composite A than for the composite D. However, this observation is mitigated by the fact that the line in composite $B$ is also quite weak. This discrepancy may be the result of the fact that very few quasars ( $\sim 10$ per composite) contribute to the composites in this region of the spectrum, thus the composite Lyman- $\alpha$ profiles (and to a lesser extent, N V) are on a much weaker footing than the other profiles discussed here.

Although more data is needed in the Lyman $\alpha+\mathrm{N}$ V region of the spectra, we note that a shift of the $\mathrm{N} V$ emission line is evident between composites A and D (see Figure 4). In composite A, the N V peak is separated from the red wing of Lyman- $\alpha$. However, in composite D, the N V emission line blends in with the red wing of Lyman- $\alpha$ - consistent with a blueshift of $\mathrm{N} \mathrm{V}$. The blending is sufficiently strong that we do not attempt to measure the velocity shift of $\mathrm{N} \mathrm{V}$; however, visual inspection of Figure 4 indicates that the N V shift may be significantly larger than found by Vanden Berk et al. (2001) - a finding which would bring N V more into line with the observed correlation between ionization potential and velocity shift (Vanden Berk et al. 2001).

Other lines, in addition to C IV, show significant changes in strength in Figure 4. Composite $\mathrm{D}$ has additional flux in the $\mathrm{Al}$ III $\lambda 1857$ line in addition to the apparent increase in Si III] flux (relative to $\mathrm{C} \mathrm{III]),} \mathrm{as} \mathrm{discussed} \mathrm{above.} \mathrm{Even} \mathrm{more} \mathrm{striking} \mathrm{is} \mathrm{the} \mathrm{almost} \mathrm{complete} \mathrm{lack} \mathrm{of} \mathrm{He} \mathrm{II}$ $\lambda 1640$ in composite D, meaning that the He II emission line region is obscured or suppressed. Alternatively, the He II flux in Composite D may simply be broader, weaker and more blueshifted than in Composite A. However, the "more blueshifted" part of this interpretation for He II is inconsistent with the C IV results, since we have argued that the C IV blueshift is not a bulk shift of the line profile to the blue, but rather a lack of red flux. Since He II is a high-ionization line, its absence in the composite with the largest shift has interesting consequences. The blend of O III], $\mathrm{Al}$ II and Fe II just redward of this line also seems to be missing. Finally, the extended Fe II emission (i.e., the $3000 \AA$ bump) appears to be similar in both composite A and D.

Perhaps as interesting as the differences in the composites in Figure 4 is one notable lack of difference: Si IV. Although Si IV is a high-ionization line and is similar in chemical structure to 
C IV, we see little difference in the Si IV profiles between the composites. There is some evidence for a shift in the line peaks, but no evidence for dearth of flux in the red wing. We have no explanation for this fact, other than to note that this finding is consistent with previous results for the Baldwin Effect (see $\S 4.2$ ), and that the ionization potential for creation of $\mathrm{C}$ IV is about equal to the ionization potential for destruction of Si IV. Thus Si IV emission will tend to come from larger radii than C IV emission, which may be a clue to the different behaviors of their profiles.

Since redshifts measured from Mg II are generally thought to be farther from systemic compared to those measured from [O III], we have also created composite spectra covering these two emission peaks for the sake of comparison. Figure 2 showed that the velocity shift of $\mathrm{Mg}$ II as compared to [O III] ranges from less than $-500 \mathrm{~km} \mathrm{~s}^{-1}$ to $500 \mathrm{~km} \mathrm{~s}^{-1}$. In Figure 8, we plot two composites of the spectra with the smallest and most negative shifts of Mg II with respect to [O III], in black, and with the largest shifts, in grey. The inset of Figure 8 shows the Mg II emission lines after the spectra have been normalized by a locally fit power-law continuum. As with the C IV profiles, the Mg II profiles are in good agreement in the blue wing with an apparent lack of flux in the red wing of the composite with the largest Mg II shift. Once again, the "shift" does not appear to be a shift of the centroid so much as a suppression of red flux in those emission lines that appear shifted. Note that without follow-up observations, we have no way of knowing if the quasars with the largest Mg II blueshifts also have the largest C IV blueshifts (although this would be a reasonable assumption).

A more thorough analysis of the emission line blueshifts would include gravitational redshifts (e.g., Popovic et al. 1995). However, we believe that they are probably not important, for a number of reasons. First, they are relatively small; using values chosen to maximize the graviational redshift ( $M_{\mathrm{BH}}=10^{9} M_{\odot}$ and a distance of 1 light-month between the black hole and the BELR) yields a gravitational reshift of only $\sim 200 \mathrm{~km} \mathrm{~s}^{-1}$ (Popovic et al. 1995). Second, unless the blueshift is correlated with the mass of the central black hole, then there should not be a correlation between the emission line blueshifts and the strength of a gravitational redshift. Finally, and most importantly, they are in the wrong direction: it is when C IV has the largest blueshift that the C IV redshift is least consistent with the redshift from narrow, forbidden emission features.

\subsection{Correlations with Other Properties}

We now turn to a comparison of the C IV velocity shift composites as a function of a number of quasar properties. Tables 1 and 2 give the properties of the four C IV shifted composite spectra. Each of the four composite spectra has a similar number of input spectra. We find that the four composites have relatively similar redshift and luminosity distributions, consistent with there being no strong correlation between these properties and the velocity shift of the C IV emission line. We

further tested this conclusion by creating luminosity and redshift composite spectra and found no significant emission line shifts between high- and low-luminosity quasars and between high- and low-redshift quasars. The redshift range covered by these composites is probably not large enough 
to draw any significant conclusions, however. On the other hand, the range in absolute magnitude, $-24.54 \geq M_{i} \geq-28.57$, is sufficient to test any significant trend with quasar luminosity. Although the distributions appear similar, there is a small trend of increasing C IV blueshift with luminosity; see $\S 4.2$ for further discussion.

We do find that there is a possible trend in the radio properties as a function of the $\mathrm{C}$ IV velocity shift. Greater C IV velocity shifts are correlated with lower radio detection fraction as can be seen in Table 2. Although the absolute numbers are small, we find that composite A has a $3 \sigma$ excess (using the statistical definitions of Gehrels 1986) of radio detections as compared to composite D. In addition, we find that there is a trend in radio flux density; the radio-detected quasars are less radio bright with increasing C IV blueshift; the four sample D have an average flux density of only $1.27 \mathrm{mJy}$. To test that our results are not biased by the inclusion of more radio sources in sample A as compared to sample D, we have also re-created the composite spectra after removing all of the radio detected quasars. We find that the removal of these spectra produces no qualitative differences between the original and non-radio composite spectra.

Unfortunately, there are not enough radio-detected quasars in our sample (only 41 of the 794 in the $\mathrm{C}$ IV/Mg II sample, many of which are only faint radio sources) to test if there is any correlation between C IV velocity shift and quasar orientation as measured by radio spectral index or radio core dominance. Nevertheless it is interesting to note that the SDSS+FIRST quasars in our sample are strongly biased against lobe-dominated quasars, since the SDSS Quasar Survey (Richards et al. 2002) requires that FIRST-selected quasars have a match to an SDSS optical source within $2^{\prime \prime}$. This restriction will cause the SDSS to fail to target (as radio-selected quasars) those objects which have strong radio lobes, but no core radio emission (many of these objects will still be targeted as color-selected quasar candidates).

Although we would expect there to be unmatched double-lobe radio sources in our sample, a search of the FIRST database out to $120^{\prime \prime}$ and an examination of the images does not reveal many cases, and there is no obvious trend with C IV blueshift. However, it may be that the high resolution of the FIRST survey causes faint lobe emission to be missed. Possible evidence in support of a lack of lobe-dominated sources is the fact that of the 18 radio-detected quasars in our sample that are brighter than $25 \mathrm{mJy}$ at $20 \mathrm{~cm}, 12$ are clearly core dominated as determined by visual inspection of the FIRST (Becker et al. 1995) images, and only one of the other six has clear radio lobes. Thus, we might draw the conclusion that there is a dearth of radio-detected quasars in sample D because there is a selection effect that causes a correlation between large C IV velocity shift and lobe-dominated quasars (which are thought to be seen edge-on to the plane of the disk). See $\S 4.5$ for further discussion.

We find a similar correlation with the X-ray detections as determined from the EDR quasar catalog (Schneider et al. 2002); however, it is attributable to the fact that quasars from composite A, for whatever reason, have more pointed ROSAT observations. There is no trend when considering only the ROSAT Faint Source Catalog sources (Voges et al. 2000). It would be interesting to 
examine the X-ray properties in more detail. Specifically, if the velocity shift effect is caused by orientation, and if the correlation between radio core-dominance and soft X-ray energy index is real (Shastri et al. 1993), then we might expect that the quasars in sample A will have flatter soft X-ray spectral indices than the quasars in sample D.

\section{Discussion}

\subsection{BELR Models}

The results presented herein will help to constrain models for the BELR, in particular, it should be clear that there cannot be any doubt as to the reality of the systematic blueshift of C IV emission lines; this shift must be accounted for in any AGN models. This could be considered a strike against the model of Elvis (2000), in which the blueshift of high-ionization emission lines relative to low-ionization emission lines has "no obvious interpretation". However, it is equally important that these models incorporate the new results herein, primarily that the blueshift does not seem to be a net blueshift, but rather a blueshift resulting from a lack of flux in the red wing of the emission line.

For cloud models, the similar blue wings of C IV emission in the different composites would appear to favor an intrinsically isotropic velocity field in an isotropically-emitting BELR ${ }^{10}$, plus obscuration that preferentially removes flux from the red wing of C IV. However, such preferential obscuration may be difficult to produce. Unfortunately, the parameter space available to BELR models is huge, and it becomes difficult to distinguish between using observations to constrain a model and fine tuning a model to match the observations ex post facto.

Thus, a detailed discussion of how our results constrain BELR models is beyond the scope of this paper. However, we briefly comment on the subject. First, we make two general assumptions: 1) that the BELR region is stratified in ionization, specifically that the low-ionization region is located farther from the continuum source than the high-ionization region (Peterson 1993), but see O'Brien et al. (1998); and 2) that an optically thick accretion disk is interior to both regions. We then hypothesize that the differences between composite A and D are an orientation effect with composite A representing a face-on configuration and composite D representing a more edge-on configuration.

Further assuming a cloud model for the BELR with a roughly isotropic distribution of outflowing clouds, one possible (but perhaps unlikely) scenario could involve an equatorial screen that is optically thin in the face-on direction, but becomes optically thick to clouds on the side of the ac-

\footnotetext{
${ }^{10}$ It has been argued that the lack of short time delay response in reverberation mapping studies appears to rule out spherically symmetric models, but this is not the case if the emission from the BEL clouds is anisotropic (Ferland et al. 1992), which is very likely.
} 
cretion disk facing away from the observer in the edge-on direction (Figure 9). The high-ionization clouds are assumed to be outflowing faster than the low-ionization clouds, and the dust layer is assumed to be located mostly interior to the typical Mg II emission radius. Such a "model" could produce emission profiles similar to those that we observe. However, if the clouds were infalling, it is difficult to imagine a way for obscuration to produce the observed results.

We must also consider disk-like BELR geometries, such as discussed by Konigl \& Kartje (1994), Bottorff et al. (1997), Murray \& Chiang (1998), and others. If we invoke a disk-wind type model instead of a spherically symmetric cloud model, then the situation may be quite different. In this case, the flow is not purely radial: the velocity differences can be due purely to orientation and a screen is not needed. However, since we observe that the emission line profiles are not simply shifted, but also they are weaker, a screen may still be necessary. For such a disk-wind model the most obvious source of a blueshift would be an outflow in the plane of the disk. In this case, a faceon orientation would yield no net shift, whereas an edge-on orientation could produce a net shift if the far side of the disk were obscured. However, we would still have to account for the fact that our observations suggest that there is not a net blueshift, just an apparent blueshift. In addition, although our hypothesis assumes that the proposed orientation effect is a result of the orientation of the disk itself, it could instead result from a change in the opening angle of the disk-wind, see $\S 4.5$ for further discussion.

We note that Murray \& Chiang (1997) were able to use a varying outer radius for the emission line gas in a disk-wind model to explain the emission profiles in Wills et al. (1993). Murray \& Chiang (1997) also found that additionally varying the inclination angle did not produce a better fit. However, the Wills et al. (1993) composites all place the peak of the C IV emission profile at zero velocity. Since the C IV emission line profiles of our four composites are so similar to theirs, it seems quite likely that their "composite 4" should be blueshifted in a similar manner to our "composite D" (and so on for the other composites). Thus, a varying outer radius cannot be the whole picture, and it would be interesting to re-address this issue.

In any case, it should be obvious that these observations and further study of both cloud and disk-wind models, combined with the results of reverberation mapping data and analysis (e.g.,

Korista et al. 1995; Done \& Krolik 1996) will lead to a significantly better understanding of the broad line region in AGN.

\subsection{The Baldwin Effect}

The Baldwin Effect was originally defined as an anti-correlation between the equivalent width of C IV emission and the continuum luminosity at $1450 \AA$ in the spectra of quasars (Baldwin 1977). While the original definition is still the dominant one, the Baldwin Effect has come to mean any anti-correlation between quasar luminosity or absolute magnitude and the strength of the broad emission lines. Since our sample shows a very clear evolution of C IV equivalent width from large 
equivalent width in sample A to a much smaller equivalent width in sample $\mathrm{D}$, a discussion of how the Baldwin Effect might relate to our results is warranted. Although the mean absolute magnitudes of our sample and a histogram of the individual absolute magnitudes in each sample (not shown) are quite similar, we were surprised to find that there is a significant difference between the samples in terms of their absolute magnitudes. The mean values of $M_{i^{*}}$ for samples $\mathrm{A}$ and $\mathrm{D}$ are -26.14 and -26.46 , respectively. This difference has a Student's $t$ (Press et al. 1992) of 4.46 with a probability of $1.05 \times 10^{-5}$, indicating a significant difference between the samples. The differences between the adjacent samples (e.g., A and B) are only marginally significant.

To determine if our results are influenced by luminosity, we have created control samples by constraining the absolute magnitude distributions for sub-samples of each sample. We first bin the absolute magnitudes in bins of $0.5 \mathrm{mag}$, then count how many quasars are in each bin for each sample. We then created a sub-sample for each sample with only the minimum number of quasars in each bin. In this way, we create sub-samples with the same absolute magnitude distributions. A Student's $t$ test confirms the similarity of the sub-samples in terms of their mean absolute $i$-band magnitudes. Using these absolute magnitude normalized subsamples, we have recreated Figure 4 and find no qualitative differences as compared to the full samples. We thus conclude that changes in $M_{i^{*}}$ are not driving the differences between the original samples.

Nevertheless, a better analysis in terms of the Baldwin Effect is still needed. Our $M_{i^{*}}$ values sample both the continuum and emission line flux at $\lambda \sim 2800 \AA$, whereas the Baldwin Effect is usually in terms of the continuum flux at $\lambda \sim 1450 \AA$. More work is clearly needed to understand the exact relationship between our observations and the Balwin Effect.

It is particularly interesting to note that the lines that show the most differences between samples A and D are also the ones that have the strongest reported Baldwin Effects, in particular, C IV and He II (Osmer, Porter, \& Green 1994). We also see no strong differences in the Si IV profiles or blueshifts; no Baldwin Effect is observed for this line (Osmer et al. 1994). These observations are unusual in the sense that both C IV and Si IV are high ionization lines with very similar chemical structure.

Finally, if the Baldwin Effect and the blueshifts of quasar emission lines are related, one must take the blueshifts into account when determining the luminosity of the quasars. Large blueshifts will cause the redshift and thus the luminosity of the quasar to be systematically underestimated. Since we have shown that those quasars with large blueshifts also have weaker C IV emission, large emission line blueshifts will cause the the Baldwin Effect to appear weaker than it should.

\subsection{Broad Absorption Line Quasars}

Since the C IV troughs in BAL quasars removes some of the C IV emission flux, it is nearly impossible to ask whether individual BAL quasars have small or large C IV emission line blueshifts. However, we can study how the ensemble average of BALs compares to the composites that we have 
created. First, we divide the BALs into two types: low-ionization (LoBALs) and high-ionization (HiBALs), where, in addition to absorption by C IV, LoBALs show absorption in Mg II and other low-ionization lines. The HiBAL composite was created by combining all of the non-LoBAL BALlike objects that were originally rejected from our C IV sample; the redshifts are all taken from $\mathrm{Mg}$ II as they were for our C IV sample. The LoBAL composite includes the LoBALs from the HiBAL sample and includes some LoBALs outside of the redshift range studied in the C IV - Mg II sample in order to improve the signal-to-noise of the composite spectrum. These BAL composite spectra were created in exactly the same manner as composites A, B, C, and D.

The composite BAL spectra were then scaled as follows. A composite spectrum (Vanden Berk et al. 2001) was fitted to each of them in the rest wavelength frame by minimizing a weighted $\chi^{2}$ merit function, normalized in the region $1725 \pm 25 \AA$. The spectral index and reddening, $\mathrm{E}(B-V)$, were allowed to vary, using a reddening law with

$$
f_{\lambda} \propto \lambda^{\alpha_{\lambda}} e^{-2 \mathrm{E}(B-V) / \lambda}
$$

(see Francis, Whiting, \& Webster 2000), where $\lambda$ is in $\mu \mathrm{m}$ and $\alpha_{\lambda}=-(2+\alpha)$. The $\chi^{2}$ function was weighted at certain wavelengths to eliminate or reduce the effects of several emission and absorption features. The weight was 0 for the major emission lines from Lyman- $\alpha$ to $\mathrm{Mg}$ II, and also for the Lyman- $\alpha$ forest; 0.5 for the region between C IV and Si IV where BAL troughs are most likely to be found; and 1 for other wavelengths. The chi-square function was then minimized using a quasi-Newton method, yielding best-fit values of the spectral index and reddening. See Reichard et al. (2002, in preparation) for a more detailed discussion.

In order to compare the BAL composites to our $\mathrm{A}, \mathrm{B}, \mathrm{C}$, and $\mathrm{D}$ composites, we also scale composites A, B, C, and D in the same manner as above. Thus the composites from samples A, B, $\mathrm{C}$, and D will have roughly the same (overall) continuum slope and shape as the BAL composites, which allows comparison of their emission line features. From Figure 10, it is quite clear that the LoBAL composite is most similar to composite D: the red wing of $\mathrm{C}$ IV matches quite well as does the $\mathrm{C}$ III] blend. In addition, the LoBAL composite also shows a clear weakening of He II emission. The HiBAL composite is most similar to composite C; the C IV and C III] emission line profiles are good matches. Also note that, based upon the upper left-hand panel, it would appear that the C IV BAL outflows begin right at zero velocity with respect to the systemic redshift (as defined by $\mathrm{Mg}$ II).

Since the viewing angles of BALs are generally thought to be constrained to a relatively small angle from the accretion disk plane, that the LoBAL composite best matches composite D is consistent with the idea that composite $\mathrm{D}$ is composed of quasars that are preferentially observed in an edge-on configuration. The fact that the HiBAL composite is most similar to composite $\mathrm{C}$ is also consistent with the orientation hypothesis: HiBALs may differ from LoBALs in that the line of sight passes through somewhat less dense gas clouds that are more highly ionized. These correlations would seem to be inconsistent with, but do not necessarily rule out, the idea that BALs may (also) be an evolutionary stage of all quasars (Becker et al. 2000). 


\subsection{Associated Absorption}

Not only does the blueshift of the C IV emission line affect the study of BAL absorption, it also affects our understanding of narrow, "associated" absorption (Foltz et al. 1986). These are absorption systems that are preferentially within $\pm 3000 \mathrm{~km} \mathrm{~s}^{-1}$ of the quasar redshift; they occur at a much higher rate (per unit redshift) than absorbers which are thought to be caused by intervening galaxies at redshifts much smaller than the quasars. Since their discovery, two hypotheses have dominated the possible explanations, Foltz et al. (1986) suggested that 1) they are due to outflows, similar to, but weaker than BALs, or 2) they are due to a virialized cluster-like velocity dispersion. However, the fact that the C IV emission line can be blueshifted changes the situation significantly (McIntosh et al. 1999).

A full analysis of this issue is beyond the scope of this work; however, we have performed a simple test that helps to shed light on this subject. One of us (GTR) examined the C IV emission line regions of all the spectra that comprise composites $\mathrm{A}$ and $\mathrm{D}$, and two samples of quasars (one each from samples A and D) with good signal-to-noise and strong associated absorption were constructed. These samples were further restricted to those objects showing the largest associated absorption redshifts (i.e., towards or in the red wing of C IV). We present the C IV emission line regions of some of the quasars from these biased sub-samples in Figure 11. The quasars on the left-hand-side are from sample A and have small C IV emission line blueshifts with respect to Mg II, whereas the quasars on the right-hand-side are from sample D and have larger C IV emission line blueshifts with respect to Mg II.

What is interesting about Figure 11 is that, in almost every case, the associated absorption is at or shortward of the systemic redshift (dashed line, as measured from $\mathrm{Mg}$ II), and thus are consistent with outflows. Large virial velocities are not necessary to explain the "infalling" absorbers. Furthermore, in only one sample A case does the absorption occur entirely in the red wing of the emission profile. For this one exception, we suspect that a C IV blueshift that has a larger error than normal is to blame; when we remeasured the Mg II redshift by hand, we found that the redshift from the automated code was too small by $\sim 900 \mathrm{~km} \mathrm{~s}^{-1}$. The expected location of C IV using the revised redshift is indicated by the dotted line in the bottom panel on the left-hand side of Figure 11. However, given the expected errors in the individual measurements, it it unlikely that the errors will produce a symmetric distribution around the C IV line center, but quite possible that the errors could explain any of the remaining apparent inflows. That is, if the velocity shifts of associated absorption lines were always referenced to the systemic redshift, we might find that associated absorbers are all outflows.

Since Foltz et al. (1986) and others (e.g., Richards et al. 2001) have found that the strong associated absorbers are predominantly found in steep-spectrum radio-loud quasars, it is interesting to think about them in terms of an orientation hypothesis. First, we note that the presence of associated absorption in our sample A quasars may be an argument against an orientation interpretation for the C IV emission line blueshifts. Steep-spectrum quasars are thought to have a 
more edge-on orientation, whereas flat-spectrum quasars are thought to be more face-on. Thus the observation that associated absorbers are predominantly found in steep-spectrum quasars and that our hypothesized face-on quasars can have strong associated absorption is apparently inconsistent.

However, it may be possible to reconcile this problem in the following manner. If we define "associated absorption" as any absorption that occurs within the profile of C IV (say within $\pm 3000 \mathrm{~km} \mathrm{~s}^{-1}$ of the emission line peak - regardless of whether the peak is blueshifted or not), and we assume that the associated absorbers are purely outflows, then we would expect to see a higher density of associated absorption per unit velocity in the objects in sample D (assuming that they are seen edge-on). This is because there is a bigger range of velocities in which the absorbers can be found in sample D quasars, since the C IV emission line is blueshifted. For example, in a quasar with no blueshift of the C IV emission line, the observable range of associated absorption velocities is only $3000 \mathrm{~km} \mathrm{~s}^{-1}$, but in a quasar where the C IV emission line is blueshifted by

$3000 \mathrm{~km} \mathrm{~s}^{-1}$, the observable range of associated absorption is $6000 \mathrm{~km} \mathrm{~s}^{-1}$; in both cases we assume that the full range over which associated absorption can be seen is $6000 \mathrm{~km} \mathrm{~s}^{-1}$, so a sample of quasars with blueshifted emission lines would be expected to have a higher density of associated absorption. If sample D objects tend to be steep-spectrum, lobe-dominated objects when they are radio sources, this could explain the excess of associated absorbers seen in steep-spectrum, lobe-dominated radio-loud quasars.

If associated absorbers are really outflows, then they may be present at velocities much larger than $3000 \mathrm{~km} \mathrm{~s}^{-1}$ from the emission redshift of the quasar, as was suggested by Richards et al. (2001). Thus, there may be a large population of narrow, intrinsic absorption systems in flatspectrum face-on quasars, see Richards et al. (2001) and references therein.

\subsection{An Orientation Effect?}

What causes the blueshifted emission lines in quasars and other similar phenomena (e.g., the Baldwin Effect)? Orientation, specifically orientation of the plane of the accretion disk is certainly one possibility. We have presented some results that are consistent with this hypothesis.

We have shown that BAL quasars are most similar to the quasars from samples $\mathrm{C}$ and D. Since most models of BALs have the BAL clouds confined to a region within a small angle of the disk (or the disk-wind), this observation may be consistent with an orientation effect. The case for associated absorption, which is preferentially seen in steep-spectrum (edge-on) quasars, is less clear, but could also be consistent with an orientation effect.

The radio properties of our sample may also support the hypothesis that orientation plays a key role in the blueshifting of the C IV emission line. We find that there are significantly more radio-detected quasars in sample $\mathrm{A}$ than sample $\mathrm{D}$ and that the sample $\mathrm{A}$ objects are brighter at radio wavelengths. If the SDSS+FIRST matching algorithm is failing to find lobe dominated quasars, or if FIRST is failing to detect some lobe dominated quasars, an orientation effect might 
explain the lack of radio-detected sources in sample D. Furthermore that the sample A sources are bright, whereas the sample D sources are faint could be the result of an orientation-related beaming effect.

An edge-on orientation for the largest C IV shifts may also be consistent with the reduced C IV emission and the lack of He II emission. These are both high-ionization lines that are likely to be formed closer to the source of ionizing radiation than low-ionization lines and thus would be easier to obscure than the low-ionization lines which are presumably formed further out. If the obscuration is restricted to the plane of the presumed accretion disk, then we might expect that some of the high-ionization lines would be completely or partially obscured in an edge-on orientation.

Furthermore, if the disk-wind model for the BELR is true, then we might expect a broadening of the lines with increasing disk inclination angle as seen in $\mathrm{H} \beta$ by Wills \& Browne (1986). Vestergaard, Wilkes, \& Barthel (2000) find that systems which appear to be inclined according to their radio properties have broader emission line wings, which is consistent with the material being seen in a preferred direction. We do indeed see an increase in the FWHM of C IV with an increase in our proposed viewing angle (see Table 2). However, as we discussed earlier, it is not clear that this broadening of the line is real; it may instead be due to partial obscuration of the line.

A possible argument against the orientation hypothesis is the fact that Baker \& Hunstead (1995) found significant differences in the strength of the $3000 \AA$ bump between composite spectra that are core- and lobe-dominated (core-dominated having stronger $3000 \AA$ bump emission). In their sample, the $3000 \AA$ bump is barely visible for steep-spectrum quasars and virtually disappears for compact steep-spectrum quasars. Since core-dominance is thought to be correlated with orientation in radio-loud quasars, this change in $3000 \AA$ bump strength may also be related to orientation. Although we do see some differences in the strength of $3000 \AA$ bump between composites A and D, they do not appear to be anywhere near as apparent as those found by Baker \& Hunstead (1995). If both our effects and the Baker \& Hunstead (1995) effects are caused by orientation, we might expect to see a bigger difference in the Fe II fluxes between composites A and D.

Although the unified model (e.g., Urry \& Padovani 1995) is well-established, more and more emphasis is being placed on properties other than orientation in terms of explaining differences between quasars. These include luminosity, the Eddington ratio, accretion rate, black hole mass, black hole spin and age (e.g., Laor 2000). For example, Boroson \& Green (1992) argue that the first eigenvector from their principal component analysis ("Eigenvector 1" — which illuminates some correlations between intrinsic quasar properties) is driven by something other than external orientation. Even though the properties of our sample are very similar to the "Eigenvector 1" properties, we prefer orientation as an explanation. However, this orientation effect need not be specific to the orientation of the accretion disk (i.e. external orientation), but rather it could be related to the opening angle of a disk wind (such as in Elvis 2000, Figure 7), where some other quasar property is causing the opening angle of the disk wind to change, thus producing an orientation type 
effect (i.e. internal orientation). A strong argument for this type of behavior (or against orientation entirely depending on how you look at it) is the intrinsic Baldwin Effect (Pogge \& Peterson 1992). Since a Baldwin type effect is seen in individual quasars as their continuum luminosity varies (but with a different slope than the "external" Baldwin Effect), the Baldwin Effect cannot be entirely due to the orientation of the accretion disk; the same is true for the emission line blueshifts if they are related to the Baldwin Effect. However, if the orientation effect is really the wind opening angle or the angle the wind makes with the disk axis and these angles can change on reasonably short timescales, then this might account for the major differences in quasar spectra.

In any case, it is certainly possible that the size of the C IV emission line blueshift may be a function of the orientation of the quasar. Whether the orientation in question is external or internal

remains to be seen. Further study, particularly with regard to radio and X-ray properties, is needed to determine if this is really the case.

\subsection{Future Work}

Some areas of future work are beyond the scope of this paper, but are clearly appropriate, including the following:

1) Ideally, we would like to know the velocity offset between the C IV and [O III] emission lines, which requires that we obtain IR spectra of the [O III] emission line region for those quasars where we already cover C IV and Mg II.

2) Similarly, we should attempt to extend our low redshift sample to cover the C IV emission line region by obtaining UV spectra of these quasars.

3) UV spectra would also be appropriate for those objects where we cannot see the Lyman- $\alpha$ emission line, since the profile of Lyman- $\alpha$ may be helpful in constraining BELR models (Kallman \& Krolik 1986).

4) If we could find a way to know how large the C IV blueshift is likely to be without looking at the C IV emission line, it would be possible to study how the C IV blueshifts correlate with broad absorption line properties.

5) A detailed study of associated absorbers among the quasars studied herein is clearly in order. In many cases, this will mean getting higher signal-to-noise spectra.

6) Since the C IV blueshifts so strongly correlate with the equivalent width of the emission lines, a more detailed investigation of how this affects the Baldwin Effect is in order.

7) It is possible that emission from Fe II and Fe III could be influencing some of the changes that we attribute to other species. Removing the iron emission complexes using the Vestergaard \& Wilkes (2001) iron template would address this issue. We have not chosen to attempt this procedure here, in part because the Vestergaard \& Wilkes (2001) iron template is not complete in the critical 
regions near C IV and Mg II emission.

8) Last, but perhaps most important, is that future AGN "models" must not fail to take into account these emission line blueshifts. Furthermore, they must be able to explain the emission line profiles. These should be key ingredients to any model.

\section{Conclusions}

Using two samples of quasars from the SDSS Early Data Release Quasar Catalog (Schneider et al. 2002), we have studied the differences in redshift of quasars as measured by different emission lines. In one sample (with 794 quasars in the redshift range, $1.54 \leq z \leq 2.2$ ), we compared the redshifts as determined from the peak of the C IV emission line to those as determined from the peak of the Mg II emission line. In the second sample (with 417 quasars in the redshift range, $0.415 \leq z \leq 0.827$ ), we compared the redshifts from Mg II to the redshifts from [O III]. Our conclusions based on these two samples can be succinctly summarizes as follows:

1) The C IV emission line is not typically found at the same redshift as Mg II, a fact which has been reported previously. The shift of C IV is typically $\sim 800 \mathrm{~km} \mathrm{~s}^{-1}$, but can be as large as $3000 \mathrm{~km} \mathrm{~s}^{-1}$.

2) This shift is apparently not a bulk shift, but rather is due to a lack of flux in the red wing of the C IV emission line profile. This fact is a new result, but is not entirely without historical precedent. Although this conclusion depends on the placement of the continuum, we feel that our handling of the continuum is appropriate and that this conclusion is robust.

3) The equivalent width of the C IV emission line decreases with increasing blueshift.

4) The FWHM of the C IV emission line increases with increasing blueshift of the peak of the emission line; however, this effect may not be real. For example, if it is true that the red wing is simply attenuated for sample D quasars, then the FWHM will not have the same meaning that it does if there is no attenuation.

5) The blueshift of the C IV emission line may correlate with the orientation of the quasar as measured by radio properties (large blueshift having an equatorial viewing angle); further work is clearly needed.

6) The apparent blueshift of the C III] emission line with increasing blueshift of the C IV emission line may instead be the result of varying flux ratios of the blended C III] and Si III] emission lines.

7) The Mg II emission line has a small blueshift with respect to [O III] in the ensemble average, but individual deviations can be as large as $\pm 500 \mathrm{~km} \mathrm{~s}^{-1}$.

8) The spectra of BAL quasars appear to be most similar to the spectra of quasars with large 
C IV emission line blueshifts. If the C IV emission line blueshift is correlated with orientation, this supports the conclusion that BALs are correlated with orientation (whether external or internal), and vice versa.

9) It is possible that redshifted "associated" absorption population may be explained by the blueshift of C IV emission (as has been suggested before). Even if there are inflows, we find no need for extremely large velocities in the "virialized" cluster material explanation.

We acknowledge helpful discussions with Mike Eracleous, Julian Krolik, Arieh Königl, Nahum Arav, Niel Brandt, Marianne Vestergaard, and Brad Peterson. We also thank Bev Wills, Michael Brotherton, and Sarah Gallagher for their comments on the manuscript. We thank the anonymous referee for suggestions that helped improve the paper, particularly for the suggestion that we include more discussion of the Baldwin Effect. GTR, TAR, and DPS acknowledge support from NSF grant AST99-00703. PBH acknowledges financial support from Chilean grant FONDECYT/1010981 and a Fundación Andes grant.

Funding for the creation and distribution of the SDSS Archive has been provided by the Alfred P. Sloan Foundation, the Participating Institutions, the National Aeronautics and Space Administration, the National Science Foundation, the U.S. Department of Energy, the Japanese Monbukagakusho, and the Max Planck Society. The SDSS Web site is http://www.sdss.org/. The Participating Institutions in the SDSS are The University of Chicago, Fermilab, the Institute for Advanced Study, the Japan Participation Group, The Johns Hopkins University, the Max-PlanckInstitute for Astronomy (MPIA), the Max-Planck-Institute for Astrophysics (MPA), New Mexico State University, Princeton University, the United States Naval Observatory, and the University of Washington. 


\section{REFERENCES}

Baker, J. C. \& Hunstead, R. W. 1995, ApJ, 452, L95

Baldwin, J. A. 1977, ApJ, 214, 679

Baldwin, J. A., Wampler, E. J., \& Gaskell, C. M. 1989, ApJ, 338, 630

Becker, R. H., White, R. L., Gregg, M. D., Brotherton, M. S., Laurent-Muehleisen, S. A., \& Arav, N. 2000, ApJ, 538, 72

Becker, R. H., White, R. L., \& Helfand, D. J. 1995, ApJ, 450, 559

Blumenthal, G. R. \& Mathews, W. G. 1975, ApJ, 198, 517

Boroson, T. A. \& Green, R. F. 1992, ApJS, 80, 109

Bottorff, M., Korista, K. T., Shlosman, I., \& Blandford, R. D. 1997, ApJ, 479, 200

Brotherton, M. S., Wills, B. J., Francis, P. J., \& Steidel, C. C. 1994, ApJ, 430, 495

Capriotti, E., Foltz, C., \& Byard, P. 1980, ApJ, 241, 903

Corbin, M. R. 1990, ApJ, 357, 346

Corbin, M. R. \& Boroson, T. A. 1996, ApJS, 107, 69

Done, C. \& Krolik, J. H. 1996, ApJ, 463, 144

Elvis, M. 2000, ApJ, 545, 63

Espey, B. R., Carswell, R. F., Bailey, J. A., Smith, M. G., \& Ward, M. J. 1989, ApJ, 342, 666

Ferland, G. J., Peterson, B. M., Horne, K., Welsh, W. F., \& Nahar, S. N. 1992, ApJ, 387, 95

Foltz, C. B., Weymann, R. J., Peterson, B. M., Sun, L., Malkan, M. A., \& Chaffee, F. H., J. 1986, ApJ, 307, 504

Francis, P. J. \& Koratkar, A. 1995, MNRAS, 274, 504

Francis, P. J., Whiting, M. T., \& Webster, R. L. 2000, Publications of the Astronomical Society of Australia, 17, 56

Fukugita, M., Ichikawa, T., Gunn, J. E., Doi, M., Shimasaku, K., \& Schneider, D. P. 1996, AJ, 111,1748

Gaskell, C. M. 1982, ApJ, 263, 79

Gehrels, N. 1986, ApJ, 303, 336 
Gunn, J. E., Carr, M., Rockosi, C., Sekiguchi, M., Berry, K., Elms, B., de Haas, E., Ivezić , Ž. ., et al., 1998, AJ, 116, 3040

Hutchings, J. B., Gower, A. C., \& Price, R. 1987, AJ, 93, 6

Kallman, T. R. \& Krolik, J. H. 1986, ApJ, 308, 805

Konigl, A. \& Kartje, J. F. 1994, ApJ, 434, 446

Korista, K. T., Alloin, D., Barr, P., Clavel, J., Cohen, R. D., Crenshaw, D. M., Evans, I. N., Horne, K., et al., 1995, ApJS, 97, 285

Krolik, J. H. 1999, Active Galactic Nuclei: From the Central Black Hole to the Galactic Environment (Princeton, New Jersey: Princeton University Press)

Laor, A. 2000, ApJ, 543, L111

McIntosh, D. H., Rix, H.-W., Rieke, M. J., \& Foltz, C. B. 1999, ApJ, 517, L73

Murray, N. \& Chiang, J. 1997, ApJ, 474, 91

—. 1998, ApJ, 494, 125

O’Brien, P. T., Dietrich, M., Leighly, K., Alloin, D., Clavel, J., Crenshaw, D. M., Horne, K., Kriss, G. A., et al., 1998, ApJ, 509, 163

Osmer, P. S., Porter, A. C., \& Green, R. F. 1994, ApJ, 436, 678

Peterson, B. M. 1993, PASP, 105, 247

—. 1997, An Introduction to Active Galactic Nuclei (Cambridge, New York: Cambridge University Press)

Pogge, R. W. \& Peterson, B. M. 1992, AJ, 103, 1084

Popovic, L. C., Vince, I., Atanackovic-Vukmanovic, O., \& Kubicela, A. 1995, A\&A, 293, 309

Press, W. H., Teukolsky, S. A., Vetterling, W. T., \& Flannery, B. P. 1992, Numerical recipes in C. The art of scientific computing (Cambridge: University Press, —c1992, 2nd ed.)

Proga, D., Stone, J. M., \& Kallman, T. R. 2000, ApJ, 543, 686

Richards, G. T., Laurent-Muehleisen, S. A., Becker, R. H., \& York, D. G. 2001, ApJ, 547, 635

Richards et al. 2002, AJ, in press

Schneider, D. P., Richards, G. T., Fan, X., Hall, P. B., Strauss, M. A., Vanden Berk, D. E., Gunn, J. E., Newberg, H. J., et al., 2002, AJ, 123, 567 
Shastri, P., Wilkes, B. J., Elvis, M., \& McDowell, J. 1993, ApJ, 410, 29

Stoughton, C., Lupton, R. H., Bernardi, M., Blanton, M. R., Burles, S., Castander, F. J., Connolly, A. J., Eisenstein, D. J., et al., 2002, AJ, 123, 485

Tytler, D. \& Fan, X. 1992, ApJS, 79, 1

Urry, C. M. \& Padovani, P. 1995, PASP, 107, 803

Vanden Berk, D. E., Richards, G. T., Bauer, A., Strauss, M. A., Schneider, D. P., Heckman, T. M., York, D. G., Hall, P. B., et al., 2001, AJ, 122, 549

Vestergaard, M. \& Wilkes, B. J. 2001, ApJS, 134, 1

Vestergaard, M., Wilkes, B. J., \& Barthel, P. D. 2000, ApJ, 538, L103

Voges, W., Aschenbach, B., Boller, T., Brauninger, H., Briel, U., Burkert, W., Dennerl, K., Englhauser, J., et al., 2000, IAU Circ., 7432, 3

Vrtilek, J. M. \& Carleton, N. P. 1985, ApJ, 294, 106

Weymann, R. 1997, in ASP Conf. Ser. 128: Mass Ejection from Active Galactic Nuclei, 3

Weymann, R. J., Morris, S. L., Foltz, C. B., \& Hewett, P. C. 1991, ApJ, 373, 23

Wilkes, B. J. 1984, MNRAS, 207, 73

Wills, B. J., Brotherton, M. S., Fang, D., Steidel, C. C., \& Sargent, W. L. W. 1993, ApJ, 415, 563

Wills, B. J. \& Browne, I. W. A. 1986, ApJ, 302, 56

York, D. G., Adelman, J., Anderson, J. E., Anderson, S. F., Annis, J., Bahcall, N. A., Bakken, J. A., Barkhouser, R., et al., 2000, AJ, 120, 1579 


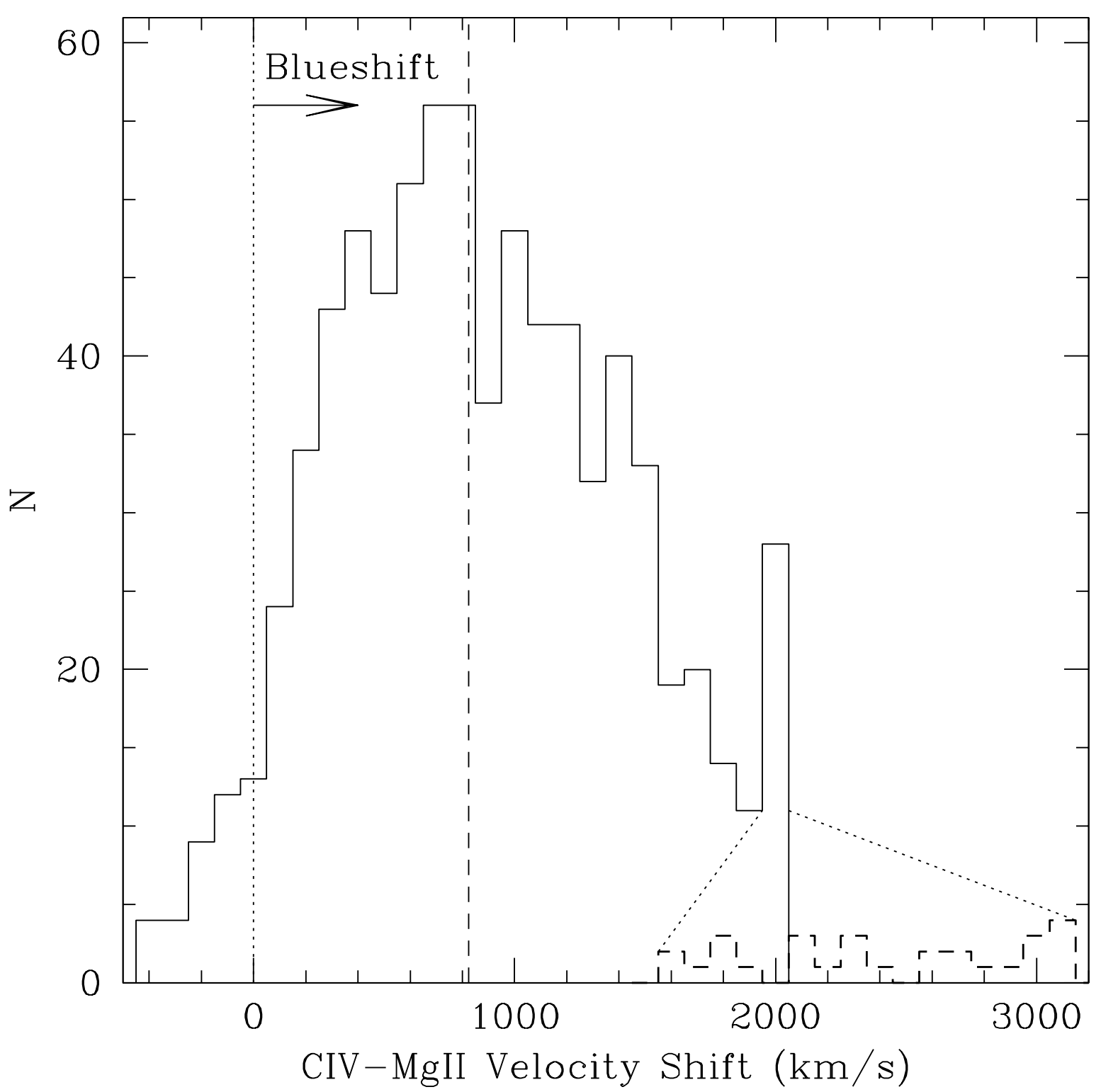

Fig. 1.- Distribution of the $794 \mathrm{C}$ IV emission line velocity shifts with respect to the $\mathrm{Mg}$ II emission line redshift (solid line). The median is $824 \mathrm{~km} \mathrm{~s}^{-1}$ (vertical, dashed line), and the dispersion is $\pm 511 \mathrm{~km} \mathrm{~s}^{-1}$. Positive velocities indicate a blueshift of C IV with respect to $\mathrm{Mg}$ II. The automated code imposes an artificial limit of $2000 \mathrm{kms}^{-1}$, which causes a pile-up at this velocity. The true range may extend as high or higher than $3000 \mathrm{kms}^{-1}$ when the shifts greater than $1950 \mathrm{~km} \mathrm{~s}^{-1}$ are recomputed by hand (28 objects; dashed histogram). Errors for individual objects can be $\gtrsim 500 \mathrm{~km} \mathrm{~s}^{-1}$, but should be much smaller in the ensemble average. The largest errors will be found in quasars with large C IV shift (because the lines are weaker, see $\S 3.2$ ) and in quasars with lower than average signal-to-noise spectra. 


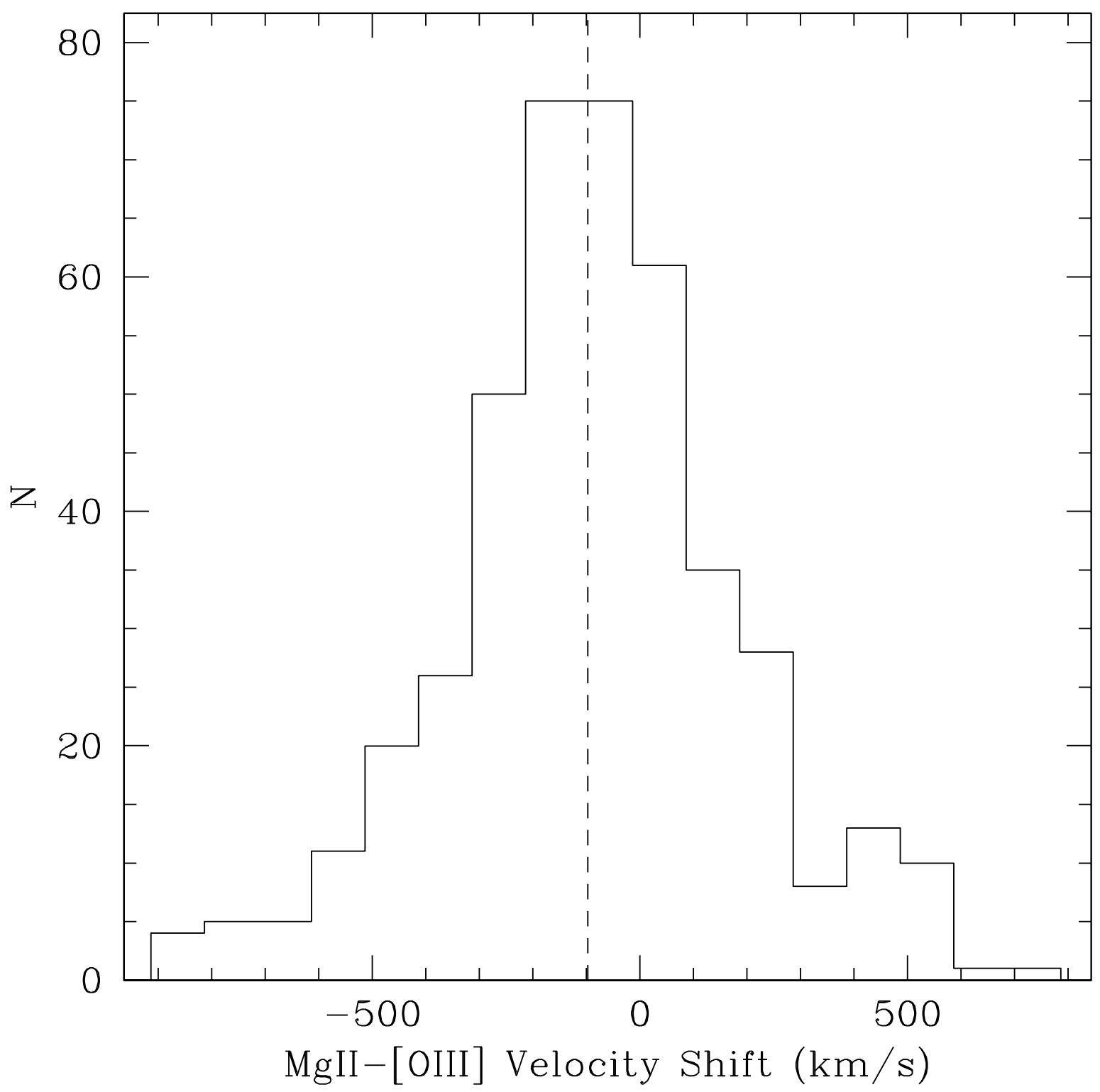

Fig. 2.- Distribution of $417 \mathrm{Mg}$ II emission line peak velocity shifts with respect to [O III]. The median is $-97 \mathrm{~km} \mathrm{~s}^{-1}$ (vertical, dashed line), and the dispersion is $\pm 269 \mathrm{~km} \mathrm{~s}^{-1}$. Positive velocities indicate a blueshift of $\mathrm{Mg}$ II with respect to [O III]. 


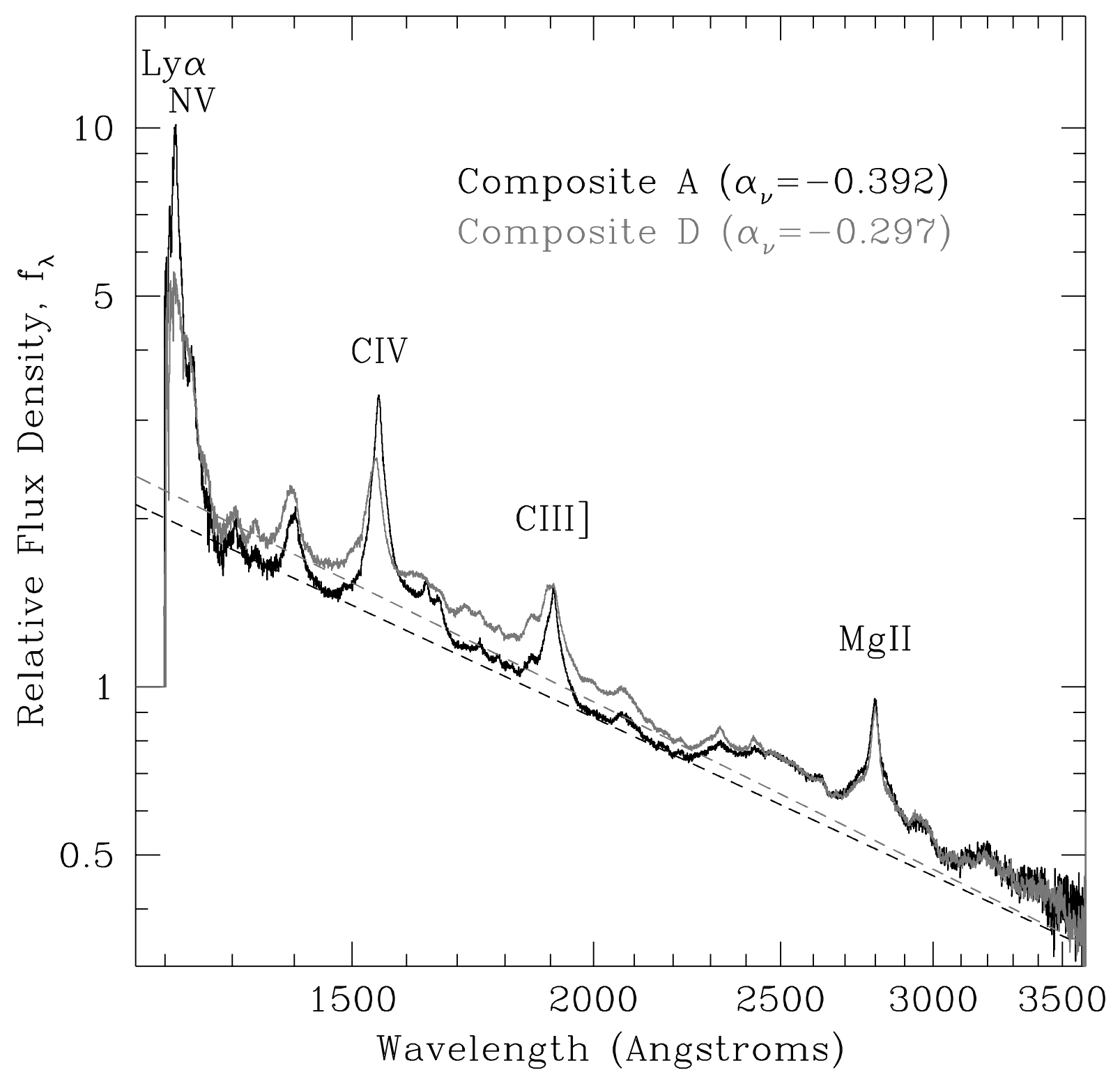

Fig. 3.- Composite spectra of 199 quasars with the smallest C IV - Mg II redshift differences (composite A, black) and 198 quasars with the largest C IV - Mg II redshift differences (composite D, grey). The spectra have not been normalized to match each other, see Vanden Berk et al. (2001) for an explanation of how the spectra are normalized internally. The power-law continua have been computed between $1355 \AA$ and $2200 \AA$ as described in the text. Major emission lines are indicated; see Vanden Berk et al. (2001) for a complete identification of the emission lines. 

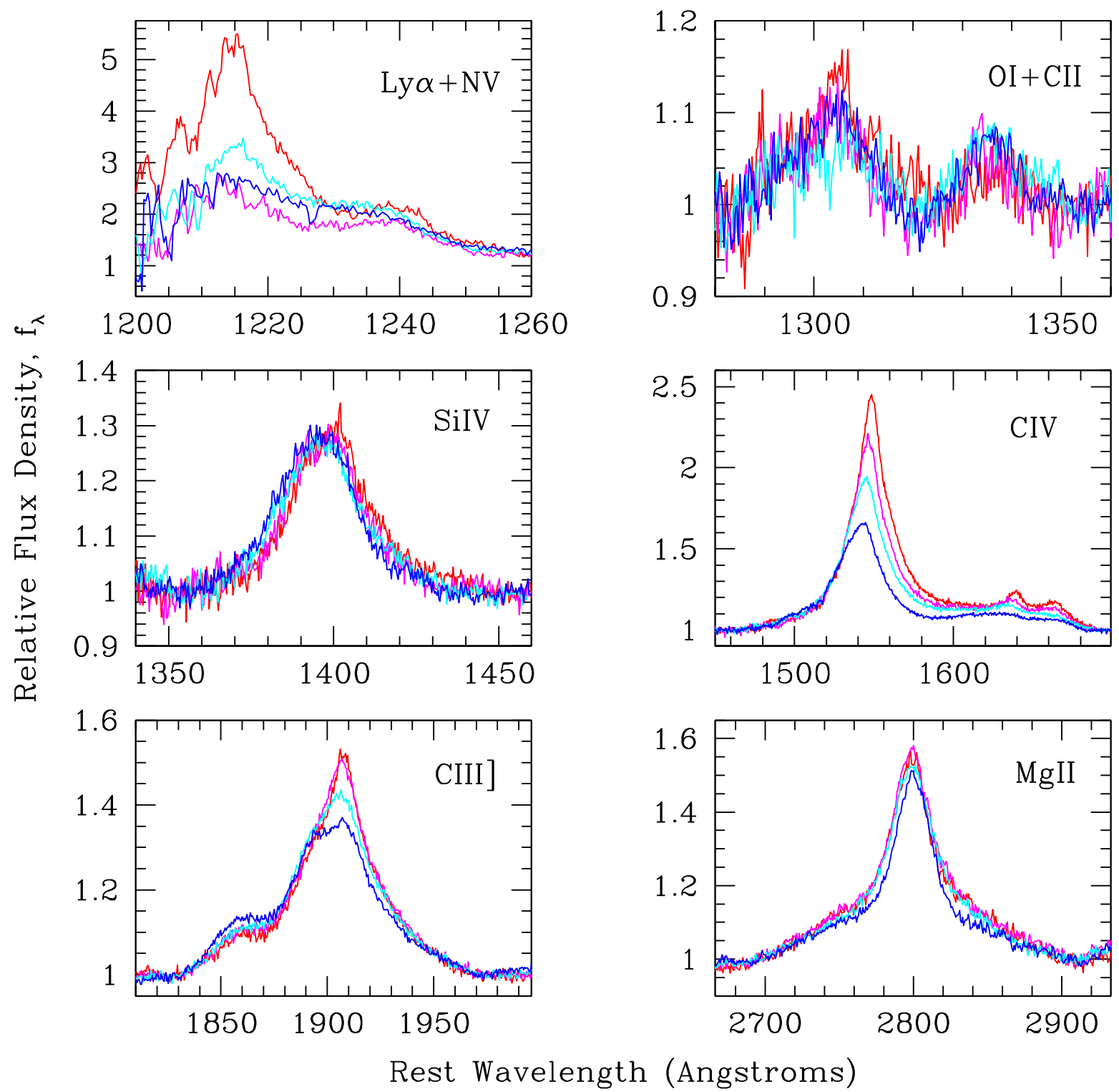

Fig. 4.- The strong emission line regions of the composite spectra, including the two intermediate C IV shift composite spectra. Local continuum regions (see text) were used instead of the global fits from Figure 3. The spectra, in the order of increasing C IV shift (composites A, B, C, and D), are plotted as red, magenta, cyan, and blue, respectively. Note the striking difference in the C IV and C III] profiles. The labels in the upper right-hand corner of each panel gives the dominant emission line(s) in each respective panel; see Vanden Berk et al. (2001) for a complete identification of the emission lines. 


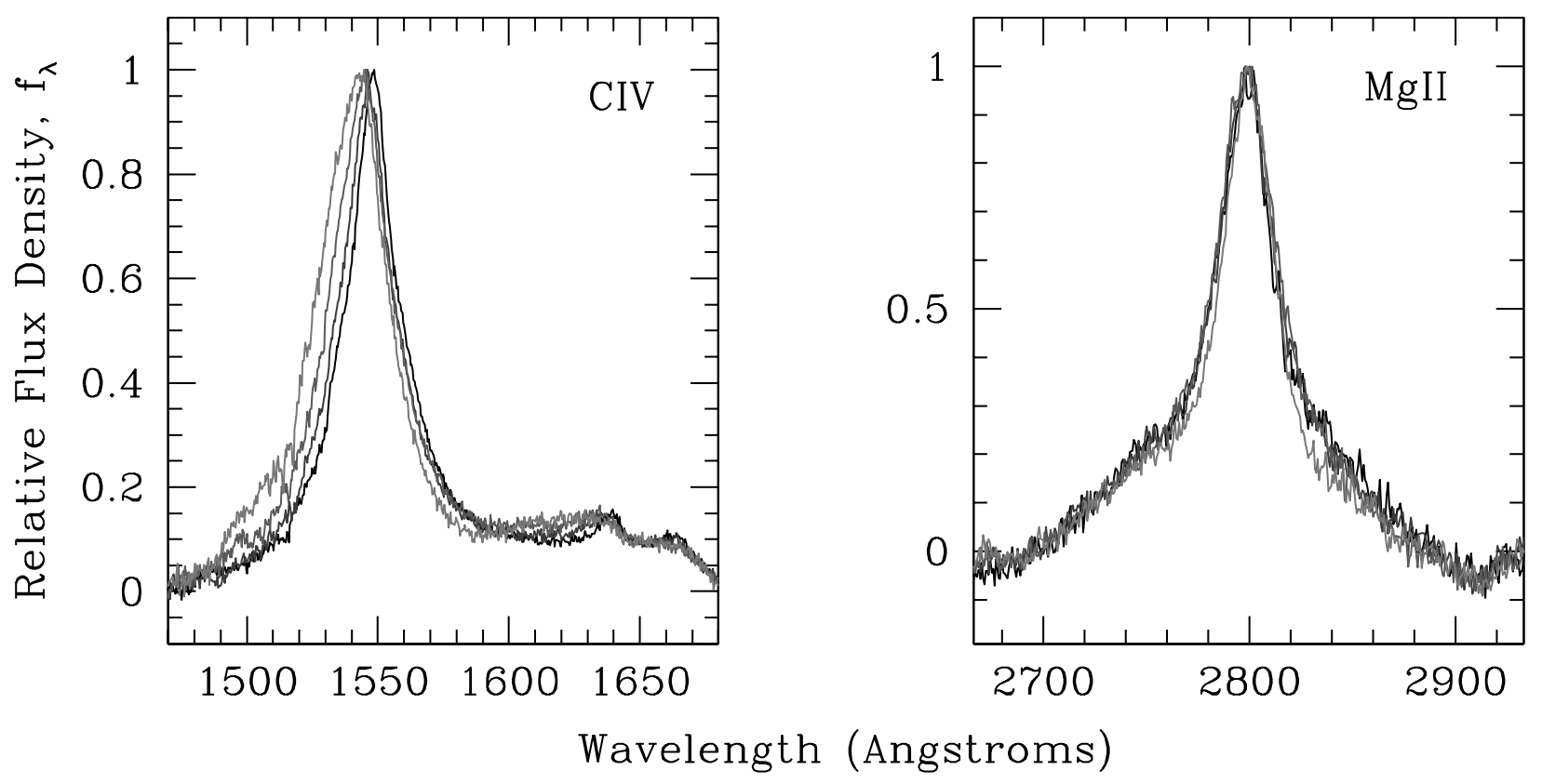

Fig. 5. - The C IV and Mg II emission line regions normalized by subtracting instead of dividing the best-fit, local, power-law continua. The peaks of the lines have also been scaled to unity so that the profiles can be compared on the same scale. Note that this scaling distorts the relative fluxes between the composites (see Figure 4); composite D has more blue flux than red as compared to composite A, but composite D has less overall blue flux. Composite A is shown in black; composite $\mathrm{D}$ is in the lightest grey. 


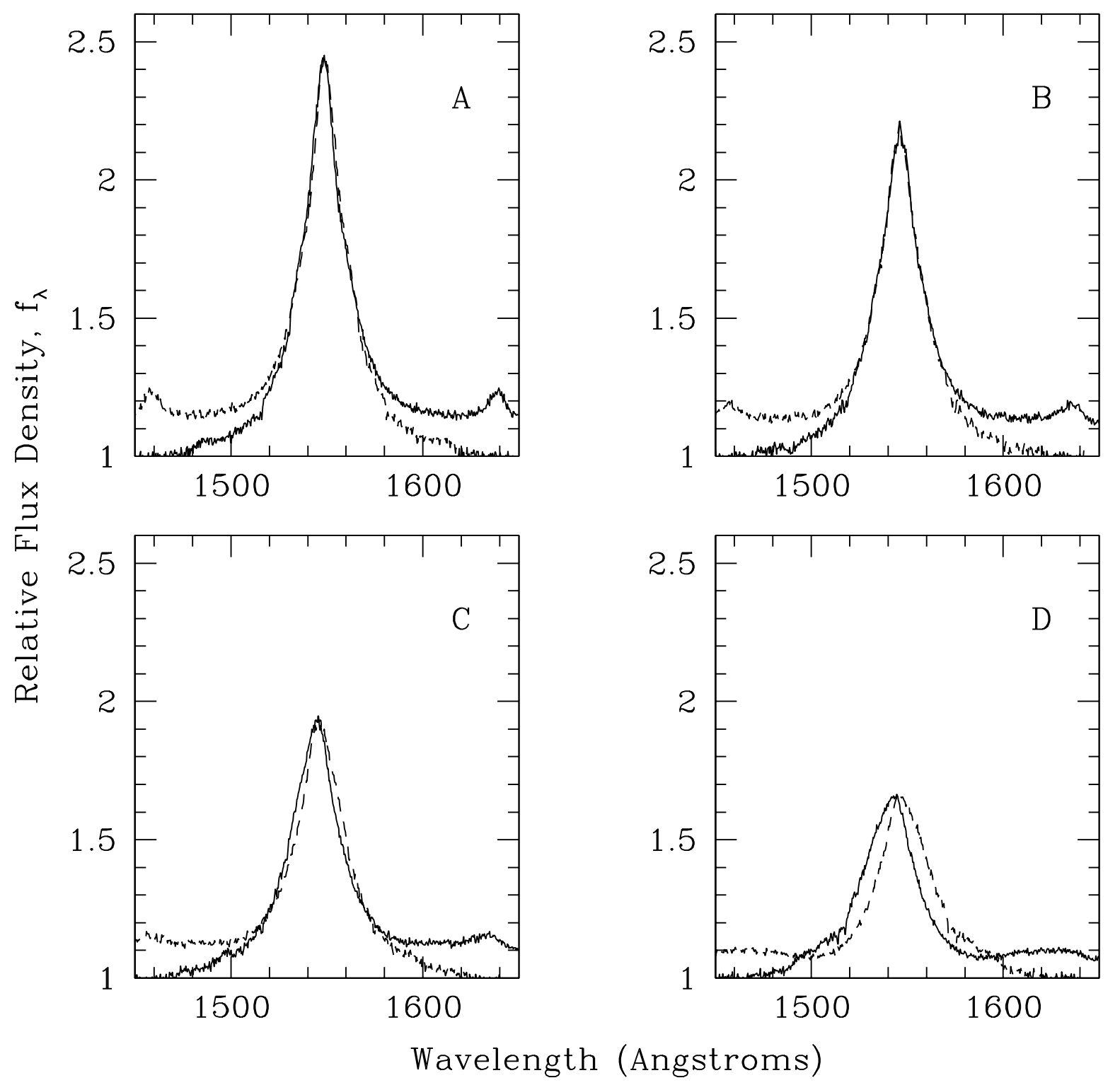

Fig. 6.- Asymmetry of the C IV profiles from composites A, B, C, and D. Each C IV profile is inverted around its peak value and is overplotted as a dashed line. Significant differences between the solid and dashed lines indicates significant asymmetry. Note that we invert around the observed peak of the line and not the expected peak, which would emphasize the asymmetries even more as a result of the apparent blueshifts of the lines. 


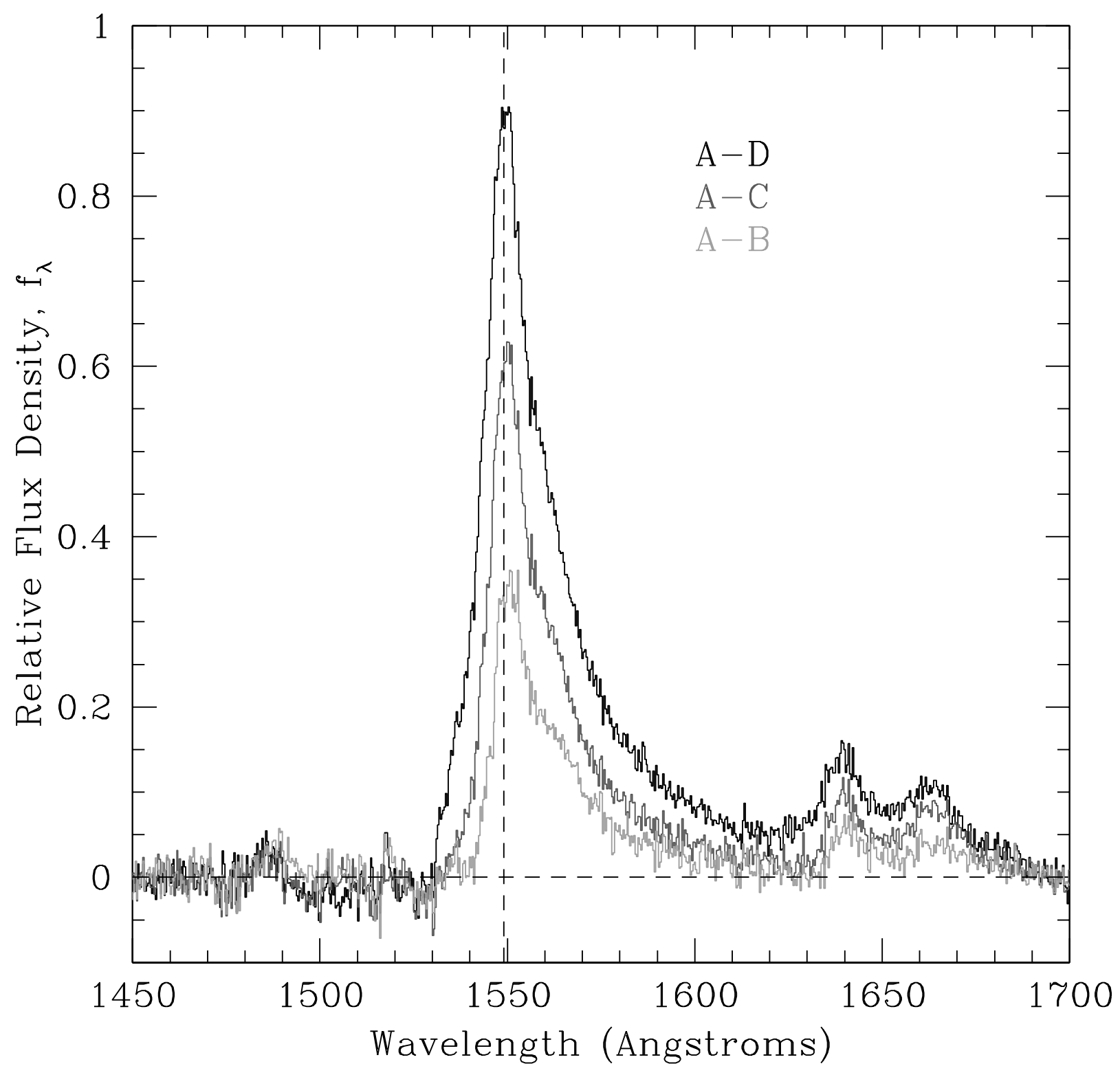

Fig. 7.- Difference spectra in the vicinity of C IV. The difference between composites A and D are shown in black, A minus C, and A minus B are shown in dark and light grey, respectively. The vertical dashed line is drawn at $1549.06 \AA$. 


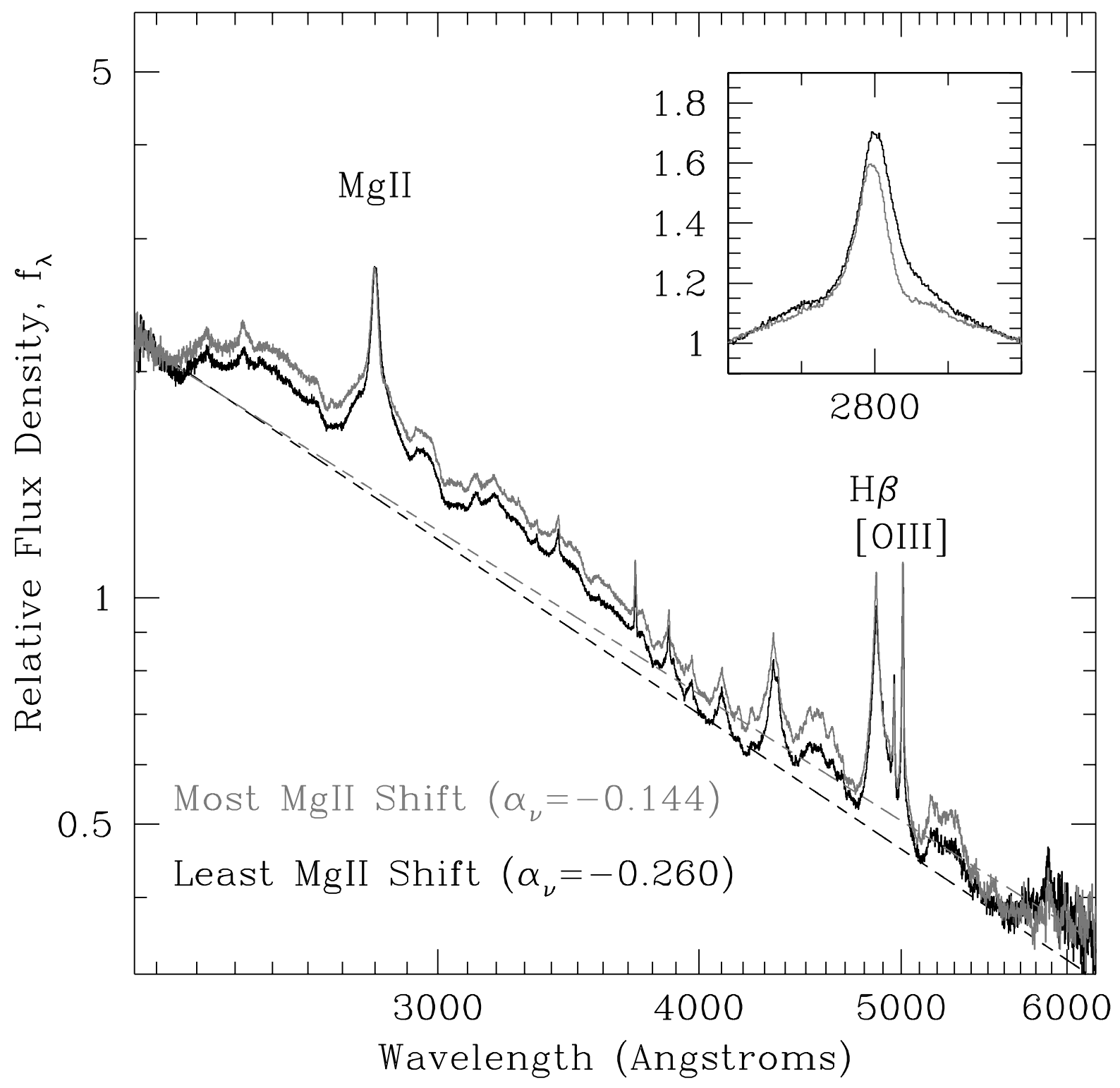

Fig. 8. - Composite spectra of quasars with large (grey) and small or negative (black) Mg II [O III] velocity shifts. Inset shows Mg II region on a linear scale after normalizing the spectra using (local) power-law continuum fits. 


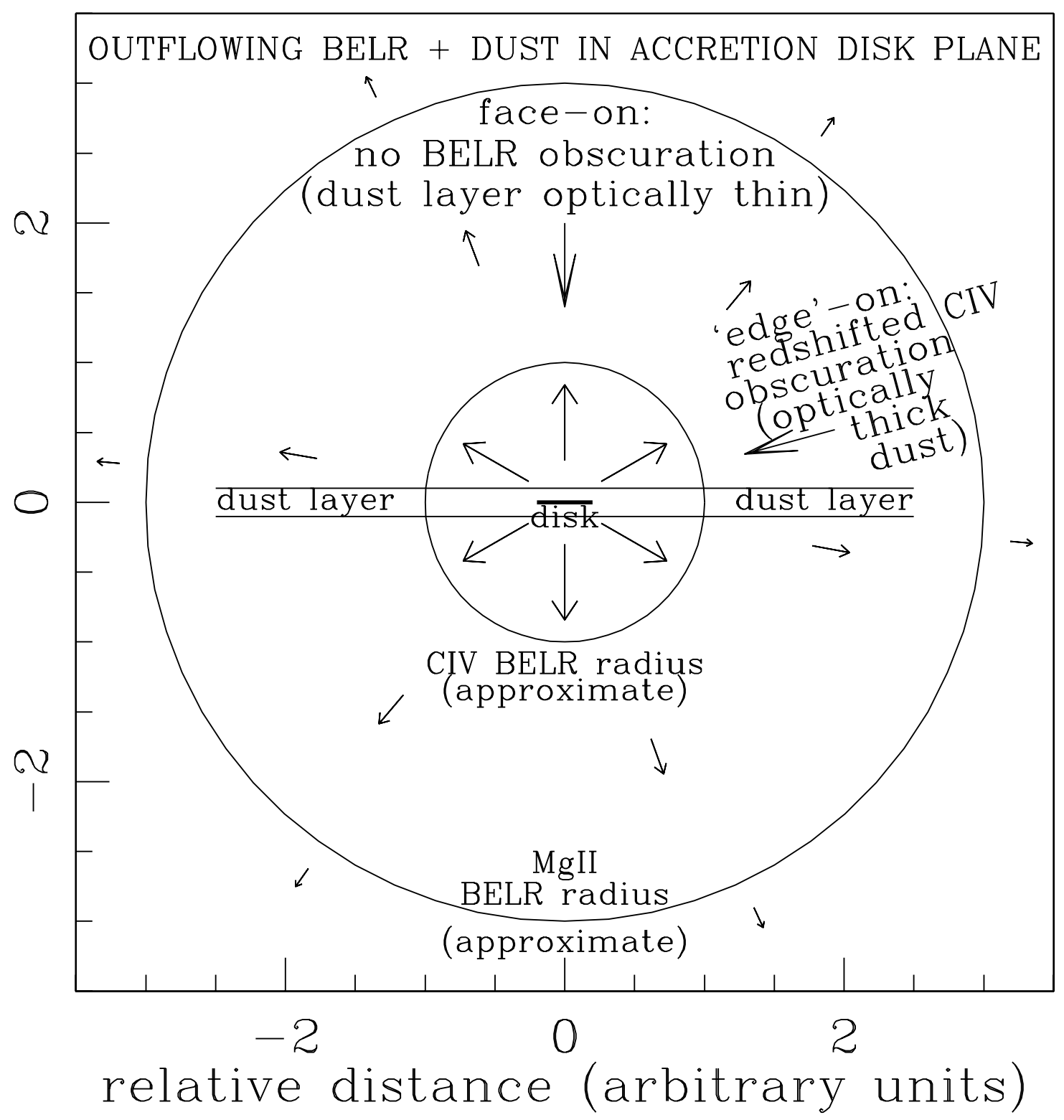

Fig. 9.- Schematic diagram of the BELR in an outflowing cloud scenario. The units are arbitrary, but the size of the C IV BELR relative to the Mg II BELR is scaled by about the same factor as the relative reverberation mapping delay times for each line (Peterson 1993). 


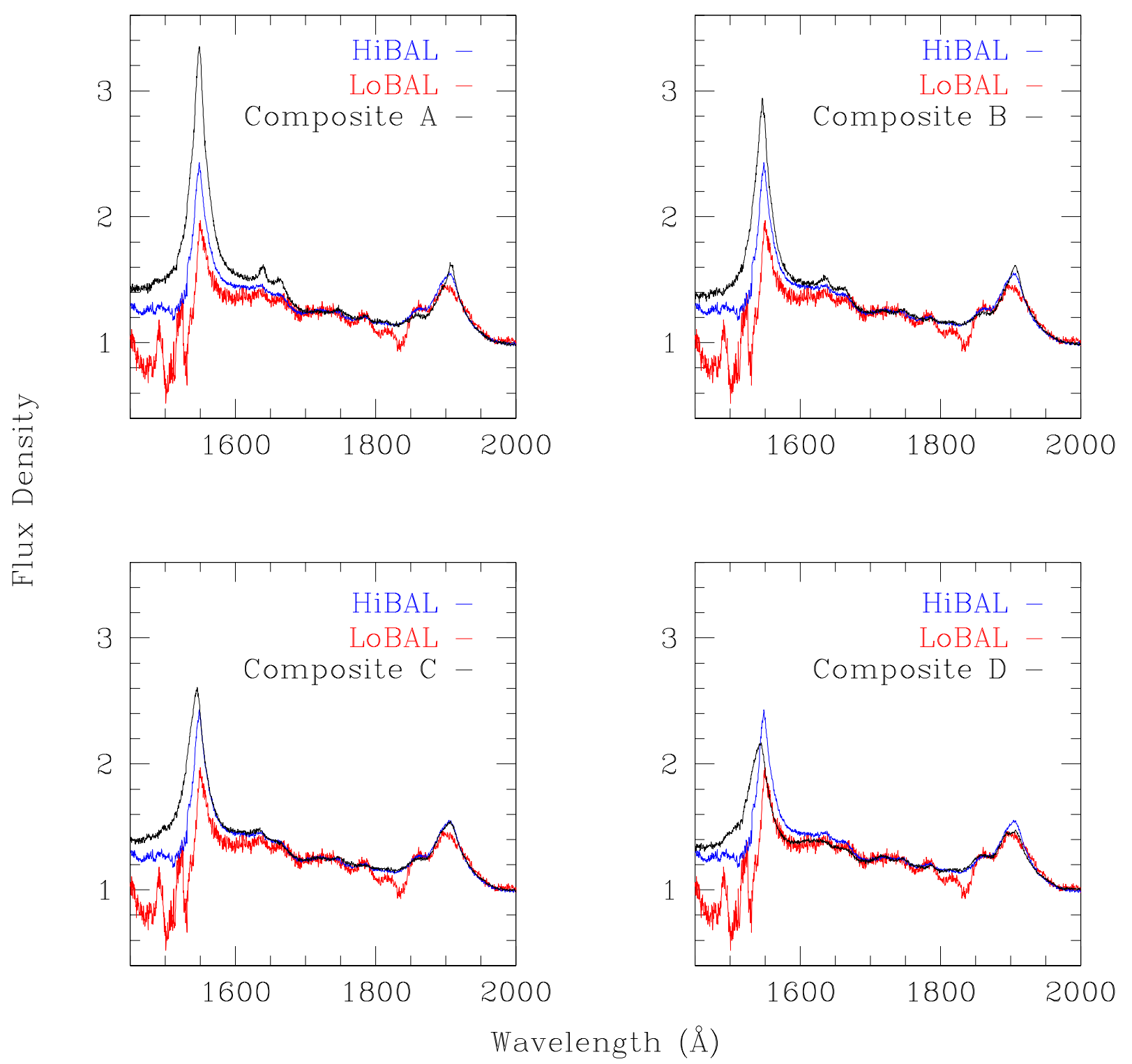

Fig. 10.- High-ionization BAL composite spectrum (HiBAL; blue line) and low-ionization BAL composite spectrum (LoBAL; red line) overlayed on each of our four composites. The C IV and C III] emission lines in the HiBAL composite most closely resemble composite C, whereas the C IV and C III] profiles in the LoBAL composite most closely resemble those in composite D. 


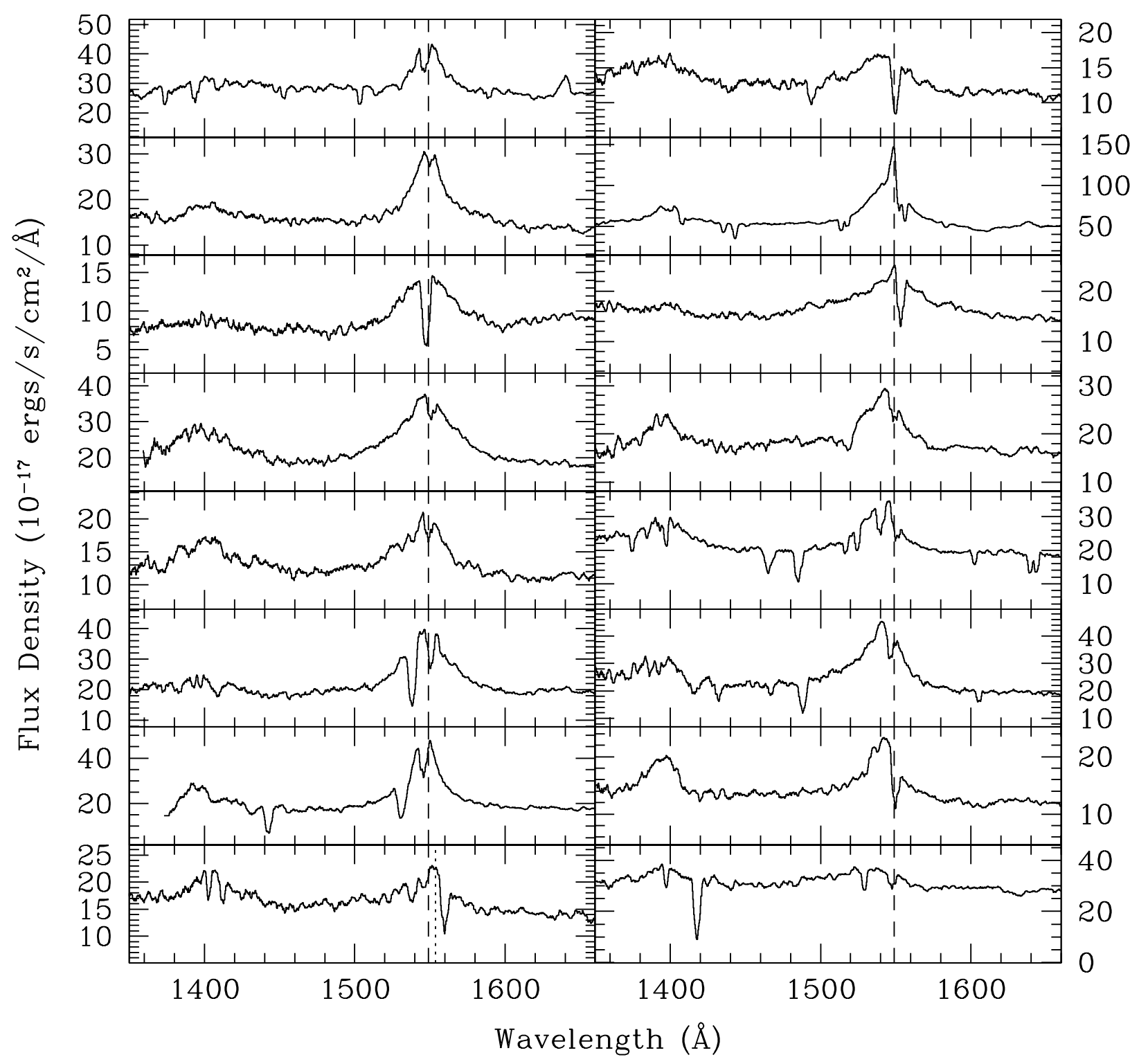

Fig. 11. - C IV emission line region of quasars with maximally redshifted ("infalling"), very strong associated absorption. Dashed line shows the expected peak of C IV based upon the Mg II redshift. (Left) Quasars from sample A, with small C IV emission line blueshifts. Dotted line in the lower left-hand panel shows the expected location of C IV based on the hand-measured Mg II redshift for this quasar. (Right) Quasars from sample D, with large C IV emission line blueshifts. 
Table 1. C IV Shift Composite Spectra Velocity Shift Data

\begin{tabular}{crrrrr}
\hline \hline Sample & $\mathrm{N}_{\mathrm{QSOs}}{ }^{\mathrm{a}}$ & $\begin{array}{c}\bar{v}_{\text {comp. }}{ }^{\mathrm{b}} \\
\left(\mathrm{km} \mathrm{s}^{-1}\right)\end{array}$ & $\begin{array}{c}\bar{v}_{\text {ind. }}{ }^{\mathrm{c}} \\
\left(\mathrm{km} \mathrm{s}^{-1}\right)\end{array}$ & $\begin{array}{c}v_{\min }{ }^{\mathrm{d}} \\
\left(\mathrm{km} \mathrm{s}^{-1}\right)\end{array}$ & $\begin{array}{c}v_{\max }{ }^{\mathrm{e}} \\
\left(\mathrm{km} \mathrm{s}^{-1}\right)\end{array}$ \\
\hline $\mathrm{A}$ & $199(160)$ & 197 & 193 & -407 & 456 \\
$\mathrm{~B}$ & $198(165)$ & 606 & 651 & 461 & 824 \\
$\mathrm{C}$ & $199(166)$ & 1003 & 1039 & 827 & 1261 \\
$\mathrm{D}$ & $198(179)$ & 1526 & 1596 & 1268 & $1993^{\mathrm{f}}$ \\
\hline
\end{tabular}

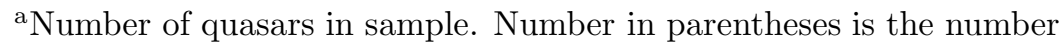
whose positions are within the FIRST survey area (Becker et al. 1995).

${ }^{\mathrm{b}}$ Average velocity offset of $\mathrm{C}$ IV with respect to $\mathrm{Mg}$ II in the composite spectrum.

${ }^{\mathrm{c}}$ Average velocity offset of $\mathrm{C} \mathrm{IV}$ with respect to $\mathrm{Mg}$ II in the individual spectra that constitute the sample.

${ }^{\mathrm{d}}$ Minimum velocity offset of $\mathrm{C}$ IV with respect to $\mathrm{Mg}$ II in the individual spectra that constitute the sample.

${ }^{\text {e}}$ Maximum velocity offset of $\mathrm{C}$ IV with respect to $\mathrm{Mg}$ II in the individual spectra that constitute the sample.

${ }^{\mathrm{f}}$ Artificial upper limit imposed by the automated analysis code; the true upper limit is $\sim 3000 \mathrm{kms}^{-1}$.

Table 2. C IV Shift Composite Spectra Properties

\begin{tabular}{cccccccc}
\hline \hline Sample & $\bar{z}$ & $\bar{M}_{i^{*}}$ & $\alpha_{\nu}$ & Radio N/\% & X - ray N/\% & C IV EQW & C IV FWHM \\
\hline A & 1.80 & -26.14 & -0.392 & $21 / 13.1$ & $4 / 2.0$ & 30.27 & 28.17 \\
B & 1.80 & -26.27 & -0.332 & $11 / 6.7$ & $8 / 4.0$ & 25.75 & 28.51 \\
C & 1.84 & -26.31 & -0.295 & $5 / 3.0$ & $3 / 1.5$ & 22.43 & 30.43 \\
D & 1.82 & -26.46 & -0.297 & $4 / 2.2$ & $2 / 2.0$ & 17.96 & 32.40 \\
\hline
\end{tabular}

aNumber and fraction of radio detections from the VLA's "FIRST" survey (Becker et al. 1995).

${ }^{b}$ Number and fraction of X-ray detections includes only the ROSAT Faint Source Catalog detections (Voges et al. 2000). 